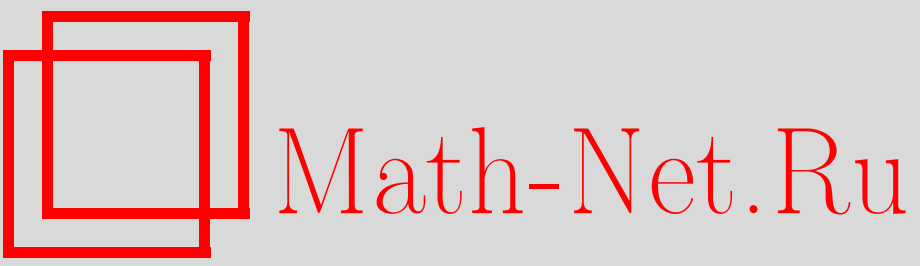

Р. Ш. Липцер, П. Чиганский, Умеренные уклонения для процесса диффузионного типа в случайной среде, Теория вероятн. и ее примен., 2009, том 54, выпуск 1, 39-62

DOI: https://doi.org/10.4213/tvp2498

Использование Общероссийского математического портала Math-Net.Ru подразумевает, что вы прочитали и согласны с пользовательским соглашением

http: //www . mathnet.ru/rus/agreement

Параметры загрузки :

IP : 54.198 .64 .247

26 апреля 2023 г., 03:07:03 
(c) 2009 г. ЛИПЦЕР Р.Ш.*, पИГАНСКИЙ П.**

\section{УМЕРЕННЫЕ УКЛОНЕНИЯ ДЛЯ ПРОЦЕССА ДИФФУЗИОННОГО ТИПА В СЛУЧАЙНОЙ СРЕДЕ}

Мы рассматриваем процесс диффузионного типа

$$
d X_{t}^{\varepsilon}=b\left(\frac{X_{t}^{\varepsilon}}{\varepsilon}\right) d t+\varepsilon^{\kappa} \sigma\left(\frac{X_{t}^{\varepsilon}}{\varepsilon}\right) d B_{t}, \quad t \leqslant T,
$$

с фиксированным начальным условием $X_{0}^{\varepsilon}=x_{0}$, броуновским движением $B_{t}$ и малым параметром $\varepsilon$. Здесь $\kappa>0-$ фиксированная положительная константа, $\sigma(u), u \in \mathbf{R}$ эргодическая стационарная марковская цепь со значениями $a_{1}, \ldots, a_{m}$ и $b(u)=g(\sigma(u))$, где $g$ измеримая ограниченная функция.

Для $\kappa<\frac{1}{6}$ мы доказываем, что семейство $\left\{X_{t}^{\varepsilon}\right\}_{\varepsilon \rightarrow 0}$ удовлетворяет принципу больших уклонений Вентцеля-Фрейдлина для диффузионного процесса с постоянными сносом и диффузией:

$$
\mathbf{b}=\sum_{i=1}^{m} \frac{g\left(a_{i}\right)}{a_{i}^{2}} \pi_{i} / \sum_{i=1}^{m} \frac{1}{a_{i}^{2}} \pi_{i}, \quad \mathbf{a}=1 / \sum_{i=1}^{m} \frac{1}{a_{i}^{2}} \pi_{i},
$$

где $\left\{\pi_{1}, \ldots, \pi_{m}\right\}$ - распределение $\sigma(0)$.

Ключевые слова и фразы: процессы диффузионного типа, случайная среда, малый параметр, принцип больших уклонений Вентцеля-Фрейдлина, умеренные уклонения.

1. Введение. В работе [4] установлен принцип больших уклонений для диффузионного векторного марковского процесса, заданного уравнением Ито и порождаемого броуновским движением $B_{t}$ :

$$
X_{t}^{\varepsilon}=x_{0}+\int_{0}^{t} b\left(\frac{X_{s}^{\varepsilon}}{\varepsilon}\right) d s+\varepsilon^{\kappa} \int_{0}^{t} \sigma\left(\frac{X_{s}^{\varepsilon}}{\varepsilon}\right) d B_{s},
$$

где $\varepsilon>0$ - малый параметр и $b(x), \sigma(x)$ - гладкие периодические функции с периодом 1.

* Department of Electrical Engineering Systems, Tel Aviv University, 69978 Tel Aviv, Israel; e-mail: liptser@eng.tau.ac.il

** Department of Statistics, The Hebrew University, Mount Scopus, Jerusalem 91905, Israel; e-mail: pchiga@mscc.huji.ac.il

1) П. Чиганский благодарит фонд Chateaubriand за поддержку этого исследования. 
В [4] показано, что имеют место три варианта принципа больших уклонений в соответствии с $\kappa \in\left(0, \frac{1}{2}\right), \kappa=\frac{1}{2}$ и $\kappa \in\left(\frac{1}{2}, \infty\right)$, где второй и третий варианты отличаются от классической версии ВентцеляФрейдлина, а первый вариант соответствует классической версии и имеет такой же функционал действия, как для семейства $\left\{\widehat{X}^{\varepsilon}\right\}_{\varepsilon \rightarrow 0}$ с постоянными сносом и диффузией. В частности, в скалярном случае

$$
\widehat{X}_{t}^{\varepsilon}=x_{0}+\mathbf{b} t+\varepsilon^{\kappa} \sqrt{\mathbf{a}} B_{t},
$$

где $\mathbf{b}=\int_{0}^{1} b(s) \sigma^{-2}(s) d s\left(\int_{0}^{1} \sigma^{-2}(s) d s\right)^{-1}$ и $\mathbf{a}=\left(\int_{0}^{1} \sigma^{-2}(s) d s\right)^{-1}$. В этом контексте, следуя терминологии из [5], будем говорить, что для $\kappa \in\left(0, \frac{1}{2}\right)$ имеет место принцип умеренных уклонений.

В этой работе мы устанавливаем принцип умеренных уклонений для скалярного процесса диффузионного типа в случайной среде, когда $b(u)=b(\omega, u)$ и $\sigma(u)=\sigma(\omega, u)$ являются случайными процессами, не зависящими от броуновского движения.

Мы предполагаем, что $\sigma(u)$ - стационарная эргодическая марковская цепь со значениями в множестве $\mathscr{A}=\left\{a_{1}, \ldots, a_{m}\right\}, a_{i} \neq 0$, с непрерывными справа и имеющими пределы слева траекториями. Мы также предполагаем, что $b(u)=g(\sigma(u))$ с некоторой ограниченной и измеримой функцией $g(x)$.

Мы доказываем существование принципа умеренных уклонений для

$$
\kappa \in\left(0, \frac{1}{6}\right) \quad \text { и } \quad \mathbf{b}=\sum_{i=1}^{m} \frac{g\left(a_{i}\right)}{a_{i}^{2}} \pi_{i} / \sum_{i=1}^{m} \frac{1}{a_{i}^{2}} \pi_{i}, \quad \mathbf{a}=1 / \sum_{i=1}^{m} \frac{1}{a_{i}^{2}} \pi_{i},
$$

где $\pi=\left(\pi_{1}, \ldots, \pi_{m}\right)$ - распределение $\sigma(0)$.

В [4] для доказательства принципа больших уклонений используется марковское свойство процесса $X_{t}^{\varepsilon}$ и асимптотический анализ (здесь и далее $\lambda(t)$ - тест-функция):

$$
\lim _{\varepsilon \rightarrow 0} \varepsilon^{2 \kappa} \ln \mathbf{E}\left(\exp \left[\frac{1}{\varepsilon^{2 \kappa}} \int_{0}^{T} \lambda(t) d X_{t}^{\varepsilon}\right]\right)
$$

(см. например, [1, гл. 2.3, 5.1], [3], [6]).

В случайной среде $X_{t}^{\varepsilon}$ уже не является марковским процессом, что чрезвычайно усложняет вычисление (1.3). Поэтому вместо (1.3) мы используем подход А. А. Пухальского и вычисляем экспоненциальный мартингал

$$
\mathfrak{z}_{T}(\lambda)=\exp \left[\frac{1}{\varepsilon^{2 \kappa}} \int_{0}^{T} \lambda(t) d X_{t}^{\varepsilon}-\ln \mathscr{E}_{T}\left(X^{\varepsilon}\right)\right],
$$

в котором кумулянта

$$
\mathscr{E}_{t}\left(X^{\varepsilon}\right)=\exp \left(\frac{1}{\varepsilon^{2 \kappa}} \int_{0}^{t}\left[\lambda(s) b\left(\frac{X_{s}^{\varepsilon}}{\varepsilon}\right)-\frac{\lambda^{2}(s)}{2} \sigma^{2}\left(\frac{X_{s}^{\varepsilon}}{\varepsilon}\right)\right] d s\right)
$$


мультипликативно компенсирует $\exp \left(\varepsilon^{-2 \kappa} \int_{0}^{T} \lambda(t) d X_{t}^{\varepsilon}\right)$ до локального мартингала $\mathfrak{z}_{t}(\lambda)$. В этом подходе достаточные условия существования принципа умеренных уклонений формулируются в терминах $\mathscr{E}_{t}\left(X^{\varepsilon}\right)$ (см. следствие 4.3 .8 в [14]). В нашей постановке задачи эти условия оказываются еще проще: в силу следствия 6.7 в [13] принцип умеренных уклонений имеет место, если найдутся константы $\mathbf{b}, \mathbf{a}>0$ и $\kappa>0$ такие, что для любого $\eta>0$

$$
\begin{aligned}
& \lim _{\varepsilon \rightarrow 0} \varepsilon^{2 \kappa} \ln \mathbf{P}\left(\sup _{t \leqslant T}\left|\int_{0}^{t}\left[b\left(\frac{X_{s}^{\varepsilon}}{\varepsilon}\right)-\mathbf{b}\right] d s\right|>\eta\right)=-\infty, \\
& \lim _{\varepsilon \rightarrow 0} \varepsilon^{2 \kappa} \ln \mathbf{P}\left(\sup _{t \leqslant T}\left|\int_{0}^{t}\left[\sigma^{2}\left(\frac{X_{s}^{\varepsilon}}{\varepsilon}\right)-\mathbf{a}\right] d s\right|>\eta\right)=-\infty ;
\end{aligned}
$$

подходящий подбор этих констант анонсирован в (1.2).

В следующем разделе обсуждается существование слабого решения стохастического уравнения (1.1) в случайной среде. Наш основной результат сформулирован в теореме 3.1 (раздел 3 ) и доказывается в разделе 5. Доказательству теоремы 3.1 предшествуют вспомогательные результаты (раздел 4). Расхождение $\kappa \in\left(0, \frac{1}{2}\right)$ и $\kappa \in\left(0, \frac{1}{6}\right)$ для осциллирующей и случайной сред обсуждается в разделе 6 .

Для удобства читателя основные детали метода Пухальского, адаптированные к нашей постановке задачи, вынесены в приложение.

2. Диффузионный процесс в случайной среде. Здесь и далее мы имеем дело со слабым решением стохастического уравнения, которое конструируется с помощью замены времени и замены вероятностной меры (по поводу других подходов см. [2], [15]).

Пусть $\sigma=(\sigma(u))_{u \in \mathbf{R}}$ - марковская цепь, определенная в предыдушем разделе, и $\beta=\left(\beta_{t}\right)_{t \geqslant 0}$ броуновское движение, не зависящее от $\sigma$. Пусть пара $(\sigma, \beta)$ задана на стохастическом базисе $(\Omega, \mathscr{F}, \mathbf{F}=$ $\left.\left(\mathscr{F}_{t}\right)_{t \geqslant 0}, \mathbf{Q}\right)$, удовлетворяющем обычным условиям. При этом $\sigma$ является $\mathscr{F}_{0}$-измеримой, а $\beta$ не зависит от $\mathscr{F}_{0}$. Для каждого $t>0$ определим момент остановки

$$
\tau_{t}=\inf \left\{r: \int_{0}^{r}\left(\varepsilon^{2 \kappa} \sigma^{2}\left(\left(\beta_{s}+x_{0}\right) / \varepsilon\right)\right)^{-1} d s \geqslant t\right\} .
$$

Поскольку выполнено неравенство $\sigma^{2}(u)>0$, то имеют место тождества $\int_{0}^{\tau_{t}}\left(\varepsilon^{2 \kappa} \sigma^{2}\left(\left(\beta_{s}+x_{0}\right) / \varepsilon\right)\right)^{-1} d s \equiv t$ и $\tau_{t} \equiv \int_{0}^{t} \varepsilon^{2 \kappa} \sigma^{2}\left(\left(\beta_{\tau_{s}}+x_{0}\right) / \varepsilon\right) d s$. Теперь введем поток $\sigma$-алгебр $\mathbf{G}=\left(\mathscr{G}_{t}\right)_{t \geqslant 0}$ с $\mathscr{G}_{t}:=\mathscr{F}_{\tau_{t}}$. Очевидно, что $\left(\beta_{\tau_{t}}, \mathscr{G}_{t}\right)$ является непрерывным мартингалом с предсказуемой квадратической характеристикой $\tau_{t}$. Тогда в силу теоремы Леви-Дуба случайный процесс

$$
B_{t}=\int_{0}^{t}\left(\varepsilon^{\kappa} \sigma\left(\left(\beta_{\tau_{s}}+x_{0}\right) / \varepsilon\right)\right)^{-1} d \beta_{\tau_{s}}
$$


является броуновским движением. Поскольку $B=\left(B_{t}\right)_{t \geqslant 0}$ не зависит от $\mathscr{F}_{0}$ и $\mathscr{F}_{0}=\mathscr{G}_{0}$, то случайный процесс $B=\left(B_{t}\right)_{t \geqslant 0}$ не зависит от $\mathscr{G}_{0}$. С другой стороны, $\sigma$ также является $\mathscr{G}_{0}$-измеримой. Поэтому $B$ и $\sigma(u)-$ независимые случайные процессы.

Обозначим $Y_{t}:=x_{0}+\beta_{\tau_{t}}$. Теперь, в силу определения $B_{t}$, имеем

$$
Y_{t}=x_{0}+\int_{0}^{t} \varepsilon^{\kappa} \sigma\left(\frac{Y_{s}}{\varepsilon}\right) d B_{s} .
$$

Итак, по крайней мере одно слабое решение уравнения (1.1) с нулевым сносом существует. Слабое решение (1.1) с ограниченным сносом легко конструируется с помощью теоремы Гирсанова. Пусть $Y_{t}$ определен в $(2.1)$, и пусть

$$
\Upsilon_{T}=\exp \left(\int_{0}^{T} \frac{b\left(Y_{s} / \varepsilon\right)}{\varepsilon^{\kappa} \sigma\left(Y_{s} / \varepsilon\right)} d B_{s}-\frac{1}{2} \int_{0}^{T} \frac{b^{2}\left(Y_{s} / \varepsilon\right)}{\varepsilon^{2 \kappa} \sigma^{2}\left(Y_{s} / \varepsilon\right)} d s\right) .
$$

Поскольку $b\left(Y_{s} / \varepsilon\right) /\left(\varepsilon^{\kappa} \sigma\left(Y_{s} / \varepsilon\right)\right)$ - ограниченный случайный процесс и $T<\infty$, имеет место равенство $\int_{\Omega} \Upsilon_{T} d \mathbf{Q}=1$. Мы определим вероятностную меру $\mathbf{P}$ полагая $d \mathbf{P}:=\mathfrak{z}_{T} d \mathbf{Q}$. Тогда по теореме Гирсанова случайный процесс

$$
\widehat{B}_{t}=B_{t}-\int_{0}^{t} \frac{b\left(Y_{s} / \varepsilon\right)}{\varepsilon^{\kappa} \sigma\left(Y_{s} / \varepsilon\right)} d s
$$

является броуновским движением относительно $(\mathbf{G}, \mathbf{P})$. Другими словами, случайный процесс $Y_{t}$, определенный на новом стохастическом базисе $\left(\Omega, \mathscr{F}, \mathbf{G}=\left(\mathscr{G}_{t}\right)_{t \geqslant 0}, \mathbf{P}\right)$, допускает следующее представление:

$$
Y_{t}=x_{0}+\int_{0}^{t} b\left(\frac{Y_{s}}{\varepsilon}\right) d s+\int_{0}^{t} \varepsilon^{\kappa} \sigma\left(\frac{Y_{s}}{\varepsilon}\right) d \widehat{B}_{s} .
$$

Таким образом, $Y_{t}$ является слабым решением уравнения (1.1) и, поскольку $\left(\widehat{B}_{t}\right)_{t \leqslant T}$ есть $\mathbf{P}$-независимым от $\mathscr{G}_{0}$, а $(\sigma(u))_{u \in \mathbf{R}}$ есть $\mathscr{G}_{0}$-измеримый процесс, процессы $\widehat{B}_{t}$ и $\sigma(u)$ являются $\mathbf{P}$-независимыми.

3. Формулировка основного результата. Пусть $X^{\varepsilon}=\left(X_{t}^{\varepsilon}\right)_{t \leqslant T}$ является слабым решением стохастического уравнения (1.1). Напомним, что $X^{\varepsilon}$ удовлетворяет принципу больших уклонений (в нашем случае умеренных уклонений) в метрическом пространстве $\left(\mathbf{C}_{[0, T]}, \varrho\right)$, где $\mathbf{C}_{[0, T]}$ - пространство непрерывных функций на $[0, T]$ и $\varrho-$ равномерная метрика, с параметром $\varepsilon^{2 \kappa}$ и функционалом действия $J(u)$, если для любых замкнутого и открытого множеств $F$ и $G$ соответственно

$$
\begin{aligned}
& \varlimsup_{\varepsilon \rightarrow 0} \varepsilon^{2 \kappa} \ln \mathbf{P}\left(X^{\varepsilon} \in F\right) \leqslant-\inf _{u \in F} J(u), \\
& \varliminf_{\varepsilon \rightarrow 0} \varepsilon^{2 \kappa} \ln \mathbf{P}\left(X^{\varepsilon} \in G\right) \geqslant-\inf _{u \in G} J(u) .
\end{aligned}
$$


Теорема 3.1. Для любого $\kappa<1 / 6$ семейство $\left\{X^{\varepsilon}\right\}_{\varepsilon \rightarrow 0}$ удовлетворяет приниипу умеренных уклонений с параметром $\varepsilon^{2 \kappa}$ и функиионалом действия

$$
J(u)= \begin{cases}\frac{1}{2 \mathbf{a}} \int_{0}^{T}\left(\dot{u}_{t}-\mathbf{b}\right)^{2} d t, & u_{0}=x_{0}, d u_{t}=\dot{u}_{t} d t, \int_{0}^{T} \dot{u}_{t}^{2} d t<\infty, \\ \infty & \text { в противном случае }\end{cases}
$$

гдe

$$
\mathbf{b}=\sum_{i=1}^{m} \frac{g\left(a_{i}\right) \pi_{i}}{a_{i}^{2}} / \sum_{i=1}^{m} \frac{\pi_{i}}{a_{i}^{2}} \quad u \quad \mathbf{a}=1 / \sum_{i=1}^{m} \frac{\pi_{i}}{a_{i}^{2}}
$$

$a\left\{\pi_{1}, \ldots, \pi_{m}\right\}$ - распределение $\sigma(0)$.

Доказательство этой теоремы использует ряд вспомогательных результатов, приведенных в следующем разделе.

4. Вспомогательные результаты. Здесь и далее: для любой матрицы $A$ через $A^{*}$ обозначается ее транспонированная матрица; для любого $x \in \mathbf{R}^{d}$ через $\operatorname{diag}(x)$ обозначается диагональная матрица с диагональю $x ; l$ - произвольная положительная константа, значение которой изменяется от случая к случаю; $\inf \{\varnothing\}=\infty$.

4.1. Разложение Пуассона. Пусть $\mathbf{F}^{\sigma}=\left(\mathscr{F}_{t}^{\sigma}\right)_{t \in \mathbf{R}}-$ поток $\sigma$ алгебр, порожденный процессом $\sigma: \mathscr{F}_{t}^{\sigma}=\{\sigma(u),-\infty<u \leqslant t\}$. Поскольку $\sigma$ является эргодической марковской цепью, ее матрица переходных интенсивностей $\Lambda$ имеет единственное нулевое собственное значение. Поэтому для любой ограниченной и измеримой функции $\Psi(x)$ с $\mathbf{E} \Psi(\sigma(0))=0$ найдется константа $\gamma>0$ такая, что $\left|\mathbf{E}\left(\Psi(\sigma(t)) \mid \mathscr{F}_{0}^{\sigma}\right)\right| \leqslant$ $l e^{-\gamma t}$ п.н. для любого $t>0$. Отсюда $\int_{0}^{\infty} \mathbf{E}\left|\mathbf{E}\left(\Psi(\sigma(t)) \mid \mathscr{F}_{0}^{\sigma}\right)\right| d t<\infty$. Тогда (см., например, $\left[12\right.$, гл. 9, §2]) процесс $\int_{0}^{t} \Psi(\sigma(s)) d s$ допускает разложение Пуассона

$$
\int_{0}^{t} \Psi(\sigma(s)) d s=V_{t}-V_{0}-M_{t}
$$

где $V_{t}$ согласован с $\mathbf{F}^{\sigma}$ и $M_{t}$ является $\mathbf{F}^{\sigma}$-мартингалом с непрерывными справа траекториями, имеющими пределы слева. В нашем случае $M_{t}-$ квадратично интегрируемый мартингал с квадратической характеристикой $\langle M\rangle_{t}$.

Лемма 4.1. 1) $\left|V_{t}\right| \leqslant l$ для любого $t \geqslant 0$.

2) $M_{t}$ - чисто разрывный квадратично интегрируемый мартингал с ограниченными скачками.

3) $d\langle M\rangle_{t}=m(t) d t, m(t) \leqslant l$.

Д о к а з а т е ль с т в о. Обозначим

$$
I(t)=\left(\begin{array}{c}
I_{\left\{\sigma(t)=a_{1}\right\}} \\
\vdots \\
I_{\left\{\sigma(t)=a_{m}\right\}}
\end{array}\right) \quad \text { и } \quad f=\left(\begin{array}{c}
\Psi\left(a_{1}\right) \\
\vdots \\
\Psi\left(a_{m}\right)
\end{array}\right) .
$$


Очевидное равенство $\Psi(\sigma(t))=f^{*} I(t)$ влечет за собой

$$
0=\mathbf{E} \Psi(\sigma(t))=f^{*} \mathbf{E} I(t)=f^{*} \pi .
$$

Это свойство $f$ и единственность нулевого собственного значения матрицы $\Lambda$ обеспечивают существование решения уравнения Пуассона

$$
\Lambda g=f
$$

которое также является единственным в классе $g^{*} \pi=0$. Впоследствии рассматриваются только решения из этого класса.

В силу леммы 9.2 в [11, гл. $9, \S 9.1]$, процесс

$$
N_{t}:=I(t)-I(0)-\int_{0}^{t} \Lambda^{*} I(s) d s
$$

является чисто разрывным мартингалом, согласованным с $\mathbf{F}^{\sigma}$, и имеет ограниченные скачки. Мы покажем, что $V_{t}=g^{*} I(t)$ и $M_{t}=g^{*} N_{t}$. Умножая слева обе части (4.3) на $g^{*}$ и принимая во внимание определения $V_{t}$ и $M_{t}$, получаем

$$
M_{t}=V_{t}-V_{0}-\int_{0}^{t} g^{*} \Lambda^{*} I(s) d s .
$$

Далее, в соответствии с (4.2), $g^{*} \Lambda^{*} I(s)=f^{*} I(s)=\Psi(\sigma(u))$.

Итак, (4.1) имеет место с $V_{t}$ и $M_{t}$, указанными выше.

Теперь утверждения 1) и 2) очевидны. Утверждение 3) доказывается следующим образом: по формуле Ито

$$
\begin{aligned}
I(t) I^{*}(t)= & I(0) I^{*}(0)+\int_{0}^{t}\left[I(s) I^{*}(s) \Lambda+\Lambda^{*} I(s) I^{*}(s)\right] d s \\
& +\int_{0}^{t}\left[I(s-) d N_{s}^{*}+d N_{s} I^{*}(s-)\right]+[N, N]_{t} \\
= & I(0) I^{*}(0)+\int_{0}^{t}\left[I(s) I^{*}(s) \Lambda+\Lambda^{*} I(s) I^{*}(s)\right] d s+\langle N\rangle_{t} \\
& + \text { мартингал, }
\end{aligned}
$$

где $\langle N\rangle_{t}$ - квадратическая вариация $N_{t}$. С другой стороны, формула Ито, примененная к тождеству $I(t) I^{*}(t) \equiv \operatorname{diag}(I(t))$, дает такое представление:

$$
I(t) I^{*}(t)=I(0) I^{*}(0)+\int_{0}^{t} \operatorname{diag}\left(\Lambda^{*} I(s)\right) d s+\text { мартингал. }
$$

Сравнивая эти представления для $I(t) I^{*}(t)$, заключаем, что предсказуемый процесс локально ограниченной вариации

$$
\int_{0}^{t}\left(\left[I(s) I^{*}(s) \Lambda+\Lambda^{*} I(s) I^{*}(s)\right]-\operatorname{diag}\left[\Lambda^{*} I(s)\right]\right) d s+\langle N\rangle_{t}
$$


с траекториями в пространстве Скорохода непрерывных справа и допускающих пределы слева функций является мартингалом с нулевой начальной точкой. Отсюда по теореме 1 в [12, гл. 2, §2] заключаем, что этот мартингал неотличим от нуля п.н. Другими словами,

$$
\langle N\rangle_{t}=\int_{0}^{t}\left(\operatorname{diag}\left[\Lambda^{*} I(s)\right]-\left[I(s) I^{*}(s) \Lambda+\Lambda^{*} I(s) I^{*}(s)\right]\right) d s \quad \text { п.н. } \quad \forall t \geqslant 0 .
$$

Следовательно, $d\langle M\rangle_{t} \equiv g^{*} d\langle N\rangle_{t} g=g^{*}\left(\operatorname{diag}\left[\Lambda^{*} I(t)\right]-\left[I(t) I^{*}(t) \Lambda+\right.\right.$ $\left.\left.\Lambda^{*} I(t) I^{*}(t)\right]\right) g d t$. Лемма 4.1 доказана.

4.2. Экспоненциальная оценка для мартингалов с ограниченными скачками. Для непрерывного мартингала $M=\left(M_{t}\right)_{t \geqslant 0} \mathrm{c}$ $M_{0}=0$ и квадратической вариацией $\langle M\rangle_{t}$ известна следующая экспоненциальная оценка (см., например, $[9$, лемма 1$])$ : для любых $q, r>0$

$$
\mathbf{P}\left(\sup _{t \leqslant T}\left|M_{t}\right| \geqslant r,\langle M\rangle_{T} \leqslant q\right) \leqslant 2 \exp \left(-\frac{r^{2}}{2 q}\right) .
$$

Подобная оценка имеет место и для разрывных мартингалов.

Лемма 4.2. Пусть $M=\left(M_{t}\right)_{t \geqslant 0}-$ чисто разрьгвный мартингал c $M_{0}=0$ и траекториями из пространства Скорохода $\mathbf{D}$ с ограниченными скачками $\left|\Delta M_{t}\right| \leqslant K$ и квадратической вариачией $\langle M\rangle_{t}$.

Тогда для любых $q, r>0$

$$
\mathbf{P}\left(\sup _{t \leqslant T}\left|M_{t}\right| \geqslant r,\langle M\rangle_{T} \leqslant q\right) \leqslant 2 \exp \left(-\frac{r^{2}}{2(K r+q)}\right) .
$$

3 а м е ч а н и е 1. При $K=0$ (4.5) превращается в (4.4).

Д о к аз а т е л ь с т в о. Обозначим $\mu=\mu(d t, d z)$ целочисленную меру, порожденную скачками $\Delta M_{t}$, и $\nu=\nu(d t, d z)$ - ее компенсатор (подробнее см., например, [12] или [7]). Поскольку $\left|\Delta M_{t}\right| \leqslant K$ и, значит, $\mu\left(\mathbf{R}_{+} \times\{|z|>K\}\right)=0$, компенсатор можно выбрать таким, чтобы выполнялось равенство $\nu\left(\mathbf{R}_{+} \times\{|z|>K\}\right)=0$. Тогда $M_{t}=\int_{0}^{t} \int_{|z| \leqslant K} z[\mu(d s, d z)-\nu(d s, d z)]$ и $\langle M\rangle_{t}=\int_{0}^{t} \int_{|z| \leqslant K} z^{2} \nu(d s, d z)$. Пусть предсказуемый процесс $\mathscr{L}_{t}(\lambda), \lambda \in \mathbf{R}$, с траекториями из пространства Скорохода $\mathbf{D}$ компенсирует $e^{\lambda M_{t}}$ до локального мартингала

$$
\mathfrak{z}_{t}(\lambda)=e^{\lambda M_{t}-\mathscr{L}_{t}(\lambda)}
$$

С помощью формулы Ито легко проверяется, что

$$
\mathscr{L}_{t}(\lambda)=G_{t}(\lambda)+\sum_{s \leqslant t}\left[\ln \left(1+\Delta G_{s}(\lambda)\right)-\Delta G_{s}(\lambda)\right],
$$

где

$$
\begin{aligned}
G_{t}(\lambda) & =\int_{0}^{t} \int_{|z| \leqslant K}\left(e^{\lambda z}-1-\lambda z\right) \nu(d s, d z), \\
\Delta G_{t}(\lambda) & =\int_{|z| \leqslant K}\left(e^{\lambda z}-1-\lambda z\right) \nu(\{t\}, d z) .
\end{aligned}
$$


Поскольку всякий положительный локальный мартингал является также супермартингалом, то $\mathbf{E}_{\mathfrak{z}_{\tau}}(\lambda) \leqslant 1$ для любого момента остановки $\tau$. Мы используем далее неравенство $\mathscr{L}_{t}(\lambda) \leqslant G_{t}(\lambda)$, вытекающее из $\Delta G_{t}(\lambda) \geqslant 0$ и $\ln (1+x)-x \leqslant 0$ для $x \geqslant 0$. В частности, отсюда следует, что $\mathfrak{z}_{\tau} \geqslant e^{\lambda M_{\tau}-G_{\tau}(\lambda)}$ для любого момента остановки $\tau$ и $\mathbf{E} e^{\lambda M_{\tau}-G_{\tau}(\lambda)} \leqslant 1$. Для $|z| \leqslant K$ и $|\lambda|<1 / K$ получаем

$$
e^{\lambda z}-\lambda z-1=\sum_{j=2}^{\infty} \frac{(\lambda z)^{j}}{j !} \leqslant \frac{\lambda^{2} z^{2}}{2} \sum_{j=0}^{\infty}|\lambda z|^{j}=\frac{\lambda^{2} z^{2}}{2} \frac{1}{1-|\lambda z|} \leqslant \frac{\lambda^{2} z^{2}}{2} \frac{1}{1-|\lambda K|} .
$$

Следовательно,

$$
G_{\tau}(\lambda) \leqslant \int_{0}^{\tau} \int_{|z| \leqslant K} \frac{\lambda^{2} z^{2}}{2} \frac{1}{1-|\lambda K|} \nu(d s, d z)=\frac{1}{1-|\lambda| K} \frac{\lambda^{2}}{2}\langle M\rangle_{\tau}
$$

и, значит, для любого измеримого множества $A$

$$
1 \geqslant \mathbf{E} I_{\{A\}} \exp \left(\lambda M_{\tau}-\frac{1}{1-|\lambda| K} \frac{\lambda^{2}}{2}\langle M\rangle_{\tau}\right) .
$$

Выбор $\tau=\inf \left\{t \leqslant T: M_{t} \geqslant r\right\}, A=\{\tau \leqslant T\} \cap\left\{\langle M\rangle_{T} \leqslant q\right\}$ и $|\lambda| \leqslant 1 / K$, а также $\left\{\sup _{t \leqslant T} M_{t} \geqslant r\right\}=\{\tau \leqslant T\}$ влечет за собой $1 \geqslant \mathbf{E} I_{\{A\}} \exp (\lambda r-$ $\left.(1-|\lambda| K)^{-1} \lambda^{2} q / 2\right)$ и далее

$$
\mathbf{P}\left(\sup _{t \leqslant T} M_{t} \geqslant r,\langle M\rangle_{T} \leqslant q\right) \leqslant \exp \left(-\frac{\lambda r(1-\lambda K)-\lambda^{2} q / 2}{1-\lambda K}\right) .
$$

Наконец, выбор $\lambda^{\prime}=\arg \max _{\lambda \in(0,1 / K)}\left[\lambda r(1-\lambda K)-\lambda^{2} q / 2\right]$ дает

$$
\mathbf{P}\left(\sup _{t \leqslant T} M_{t} \geqslant r,\langle M\rangle_{T} \leqslant q\right) \leqslant \exp \left(-\frac{r^{2}}{2(K r+q)}\right) .
$$

Заметим, что такое же неравенство имеет место и для $\sup _{t \leqslant T}\left(-M_{t}\right)$. Теперь (4.5) можно вывести с помощью $\mathbf{P}(A \cup B) \leqslant 2[\mathbf{P}(A) \vee \mathbf{P}(B)]$. Лемма 4.2 доказана.

5. Доказательство теоремы 3.1. Напомним, что (1.4) обеспечивает принцип умеренных уклонений. Мы начнем с проверки первого условия в (1.4). Введем стационарный процесс

$$
\theta(t)=\frac{b(t)-\mathbf{b}}{\sigma^{2}(t)}
$$

где $\mathbf{b}$ - такая константа, что $\mathbf{E} \theta(t)=0$, т.е. $\mathbf{b}=\sum_{i=1}^{m}\left(g\left(a_{i}\right) \pi_{i} / a_{i}^{2}\right) \times$ $\left(\sum_{i=1}^{m} \pi_{i} / a_{i}^{2}\right)^{-1}$. Положим

$$
H(x)=\int_{0}^{x} \int_{0}^{v} \theta(s) d s d v .
$$


Случайная функция $H(x)$ имеет непрерывную первую производную и ограниченную вторую соболевскую производную. Это позволяет применить версию Крылова формулы Ито (см. [8]) и получить

$H\left(\frac{X_{t}^{\varepsilon}}{\varepsilon}\right)=H\left(\frac{x_{0}}{\varepsilon}\right)+\frac{1}{\varepsilon} \int_{0}^{t} \int_{0}^{X_{v}^{\varepsilon} / \varepsilon} \theta(s) d s d X_{v}^{\varepsilon}+\frac{1}{\varepsilon^{2(1-\kappa)}} \int_{0}^{t}\left[b\left(\frac{X_{s}^{\varepsilon}}{\varepsilon}\right)-\mathbf{b}\right] d s$.

Отсюда

$$
\begin{aligned}
\int_{0}^{t}[ & \left.b\left(\frac{X_{s}^{\varepsilon}}{\varepsilon}\right)-\mathbf{b}\right] d s=\varepsilon^{2(1-\kappa)} \int_{0}^{X_{t}^{\varepsilon} / \varepsilon} \int_{0}^{v} \theta(s) d s d v \\
& -\varepsilon^{2(1-\kappa)} \int_{0}^{x_{0} / \varepsilon} \int_{0}^{v} \theta(s) d s d v-\varepsilon^{1-2 \kappa} \int_{0}^{t} \int_{0}^{X_{v}^{\varepsilon} / \varepsilon} \theta(s) d s b\left(\frac{X_{v}^{\varepsilon}}{\varepsilon}\right) d v \\
& -\varepsilon^{1-\kappa} \int_{0}^{t} \int_{0}^{X_{v}^{\varepsilon} / \varepsilon} \theta(s) d s \sigma\left(\frac{X_{v}^{\varepsilon}}{\varepsilon}\right) d B_{v} .
\end{aligned}
$$

Пусть $Z_{t}^{\varepsilon}$ обозначает любое слагаемое в правой части (5.1). Покажем, что

$$
\lim _{\varepsilon \rightarrow 0} \varepsilon^{2 \kappa} \ln \mathbf{P}\left(\sup _{t \leqslant T}\left|Z_{t}^{\varepsilon}\right|>\eta\right)=-\infty .
$$

Для упрощения доказательства (5.2) покажем, что множество $\Upsilon_{C}^{\varepsilon}=$ $\left\{\sup _{t \leqslant T}\left|X_{t}^{\varepsilon}\right|>C\right\}$ является экспоненциально пренебрежимым в том смысле, что

$$
\lim _{C \rightarrow \infty} \varlimsup_{\varepsilon \rightarrow 0} \varepsilon^{2 \kappa} \ln \mathbf{P}\left(\Upsilon_{C}^{\varepsilon}\right)=-\infty .
$$

Обозначим $A_{t}^{\varepsilon}=x_{0}+\int_{0}^{t} b\left(X_{s}^{\varepsilon} / \varepsilon\right) d s$ и $M_{t}^{\varepsilon}=\varepsilon^{\kappa} \int_{0}^{t} \sigma\left(X_{s}^{\varepsilon} / \varepsilon\right) d B_{s}$. Поскольку $X_{t}^{\varepsilon}=A_{t}^{\varepsilon}+M_{t}^{\varepsilon}$ и случайная величина $\sup _{t \leqslant T}\left|A_{t}^{\varepsilon}\right|$ ограничена константой, не зависящей от $\varepsilon$, то доказательство (5.3) сводится к доказательству

$$
\lim _{C \rightarrow \infty} \varlimsup_{\varepsilon \rightarrow 0} \varepsilon^{2 \kappa} \ln \mathbf{P}\left(\sup _{t \leqslant T}\left|M_{t}^{\varepsilon}\right|>C\right)=-\infty .
$$

Использование $\mathbf{P}\left(\left\langle M^{\varepsilon}\right\rangle_{T} \leqslant l T \varepsilon^{2 \kappa}\right)=1$ и (4.4) позволяет установить верхнюю оценку $\mathbf{P}\left(\sup _{t \leqslant T}\left|M_{t}^{\varepsilon}\right|>C\right) \leqslant 2 \exp \left(-C^{2} /\left(2 \varepsilon^{2 \kappa} l T\right)\right)$ и, значит, (5.3) выполнено. Ввиду (5.3) можно доказательство (5.2) заменить доказательством

$$
\varlimsup_{\varepsilon \rightarrow \infty} \varepsilon^{2 \kappa} \ln \mathbf{P}\left(\sup _{t \leqslant T}\left|Z_{t}^{\varepsilon}\right| \geqslant \eta, \Omega \backslash \Upsilon_{C}^{\varepsilon}\right)=-\infty \quad \forall C>0 .
$$

Пусть $Z_{t}^{\varepsilon}:=\varepsilon^{2(1-\kappa)} \int_{0}^{X_{t}^{\varepsilon} / \varepsilon} \int_{0}^{v} \theta(s) d s d v$. Тогда на множестве $\left\{\Omega \backslash \Upsilon_{C}^{\varepsilon}\right\}$ случайная величина $\sup _{t \leqslant T}\left|Z_{t}^{\varepsilon}\right|$ ограничена сверху случайной величиной $C \sup _{|v| \leqslant C}\left|\varepsilon^{1-2 \kappa} \int_{0}^{v / \varepsilon} \theta(s) d s\right|$. Следовательно,

$$
\begin{aligned}
\mathbf{P}\left(\sup _{t \leqslant T}\left|Z_{t}^{\varepsilon}\right| \geqslant \eta, \Omega \backslash \Upsilon_{C}^{\varepsilon}\right) & \leqslant \mathbf{P}\left(\sup _{|v| \leqslant C}\left|\varepsilon^{1-2 \kappa} \int_{0}^{v / \varepsilon} \theta(s) d s\right| \geqslant \eta, \Omega \backslash \Upsilon_{C}^{\varepsilon}\right) \\
& \leqslant \mathbf{P}\left(\sup _{|v| \leqslant C}\left|\varepsilon^{1-2 \kappa} \int_{0}^{v / \varepsilon} \theta(s) d s\right| \geqslant \eta\right)
\end{aligned}
$$


и, значит, (5.4) имеет место при условии, что

$$
\lim _{\varepsilon \rightarrow 0} \varepsilon^{2 \kappa} \ln \mathbf{P}\left(\sup _{|v| \leqslant C}\left|\varepsilon^{1-2 \kappa} \int_{0}^{v / \varepsilon} \theta(s) d s\right| \geqslant \eta\right)=-\infty .
$$

Для $Z_{t}^{\varepsilon}:=\varepsilon^{1-2 \kappa} \int_{0}^{t} \int_{0}^{X_{v}^{\varepsilon} / \varepsilon} \theta(s) d s b\left(X_{v}^{\varepsilon} / \varepsilon\right) d v$ и $Z_{t}^{\varepsilon}:=\varepsilon^{2(1-\kappa)} \int_{0}^{x_{0} / \varepsilon} \int_{0}^{v} \theta(s) d s d v$ условие (5.5) также влечет за собой (5.4).

Напомним, что $\theta(s)$ - стационарный процесс в узком смысле и, значит, распределения случайных величин

$$
\sup _{0 \leqslant v \leqslant C}\left|\int_{0}^{v / \varepsilon} \theta(s) d s\right| \text { и } \sup _{0 \leqslant v \leqslant C}\left|\int_{-v / \varepsilon}^{0} \theta(s) d s\right|
$$

совпадают. Следовательно,

$$
\begin{aligned}
\mathbf{P}\left(\sup _{|v| \leqslant C}\left|\varepsilon^{1-2 \kappa} \int_{0}^{v / \varepsilon} \theta(s) d s\right| \geqslant \eta\right) \leqslant & 2\left[\mathbf{P}\left(\sup _{0 \leqslant v \leqslant C}\left|\varepsilon^{1-2 \kappa} \int_{0}^{v / \varepsilon} \theta(s) d s\right| \geqslant \frac{\eta}{2}\right)\right. \\
& \left.\vee \mathbf{P}\left(\sup _{0 \leqslant v \leqslant C}\left|\varepsilon^{1-2 \kappa} \int_{-v / \varepsilon}^{0} \theta(s) d s\right| \geqslant \frac{\eta}{2}\right)\right] \\
= & 2 \mathbf{P}\left(\sup _{0 \leqslant v \leqslant C}\left|\varepsilon^{1-2 \kappa} \int_{0}^{v / \varepsilon} \theta(s) d s\right| \geqslant \frac{\eta}{2}\right) .
\end{aligned}
$$

Таким образом, доказательство (5.5) можно заменить доказательством

$$
\lim _{\varepsilon \rightarrow 0} \varepsilon^{2 \kappa} \ln \mathbf{P}\left(\sup _{0<v \leqslant C}\left|\varepsilon^{1-2 \kappa} \int_{0}^{v / \varepsilon} \theta(s) d s\right| \geqslant \frac{\eta}{2}\right)=-\infty .
$$

Использование $\theta(s)=\Psi(\sigma(s)), \mathbf{E} \theta(s)=0$ и леммы 4.1 обеспечивает разложение Пуассона

$$
\varepsilon^{1-2 \kappa} \int_{0}^{v / \varepsilon} \theta(s) d s=\varepsilon^{1-2 \kappa}\left[V_{v / \varepsilon}-V_{0}\right]+\varepsilon^{1-2 \kappa} M_{v / \varepsilon},
$$

где $V_{t}$ - ограниченный процесс, а $M_{t}$ - чисто разрывный мартингал с ограниченными скачками $\left|\Delta M_{s}\right| \leqslant l$ и $\langle M\rangle_{v} \leqslant l v$. Поэтому достаточно доказать, что для любого $\eta>0$

$$
\lim _{\varepsilon \rightarrow 0} \varepsilon^{2 \kappa} \ln \mathbf{P}\left(\sup _{0<v \leqslant C}\left|\varepsilon^{1-2 \kappa} M_{v / \varepsilon}\right| \geqslant \eta\right)=-\infty .
$$

В соответствии с $(4.5)$ и $\mathbf{P}\left(\left\langle\varepsilon^{1-2 \kappa} M\right\rangle_{C / \varepsilon} \leqslant l C \varepsilon^{1-4 \kappa}\right)=1$, для $\kappa<\frac{1}{4}$ имеет место следующая оценка:

$$
\mathbf{P}\left(\sup _{0 \leqslant v \leqslant C}\left|\varepsilon^{1-2 \kappa} M_{v / \varepsilon}\right| \geqslant \eta\right) \leqslant 2 \exp \left(-\frac{\eta^{2}}{2\left(l \eta \varepsilon^{1-2 \kappa}+l C \varepsilon^{1-4 \kappa}\right)}\right) .
$$

Отсюда для $\kappa<\frac{1}{6}$ получаем неравенство

$$
\varepsilon^{2 \kappa} \ln \mathbf{P}\left(\sup _{0 \leqslant v \leqslant C}\left|\varepsilon^{1-2 \kappa} M_{v / \varepsilon}\right| \geqslant \eta\right) \leqslant \varepsilon^{2 \kappa} \ln 2-\frac{\eta^{2}}{\varepsilon^{1-6 \kappa} 2 l\left(\eta \varepsilon^{2}+C\right)},
$$


Умеренные уклонения в случайной среде

11

т.е. (5.7) выполняется.

Итак, нам осталось проверить (5.2) для $Z_{t}^{\varepsilon}:=\varepsilon^{1-\kappa} \int_{0}^{t} \int_{0}^{X_{v}^{\varepsilon} / \varepsilon} \theta(s) d s \times$ $\sigma\left(X_{v}^{\varepsilon} / \varepsilon\right) d B_{v}$. Заметим, что $Z_{t}^{\varepsilon}$ - непрерывный мартингал с $\left\langle Z^{\varepsilon}\right\rangle_{t}=$ $\varepsilon^{2(1-\kappa)} \int_{0}^{t}\left(\int_{0}^{X_{v}^{\varepsilon} / \varepsilon} \theta(s) d s\right)^{2} \sigma^{2}\left(X_{v}^{\varepsilon} / \varepsilon\right) d v$. Ввиду $\sigma^{2}(u) \leqslant l$ имеет место оценка

$$
\left\langle Z^{\varepsilon}\right\rangle_{T} \leqslant T l \sup _{|v| \leqslant C}\left(\varepsilon^{1-\kappa} \int_{0}^{v / \varepsilon} \theta(s) d s\right)^{2} \quad \text { на множестве } \Omega \backslash \Upsilon_{C}^{\varepsilon} .
$$

Для $r>0$ пишем

$$
\begin{aligned}
\mathbf{P}\left(\sup _{t \leqslant T}\left|Z_{t}^{\varepsilon}\right|>\eta, \Omega \backslash \Upsilon_{C}^{\varepsilon}\right) & \\
\leqslant & \mathbf{P}\left(\sup _{t \leqslant T}\left|Z_{t}^{\varepsilon}\right|>\eta,\left\langle Z^{\varepsilon}\right\rangle_{T} \leqslant T l \sup _{|v| \leqslant C}\left|\varepsilon^{1-\kappa} \int_{0}^{v / \varepsilon} \theta(s) d s\right|^{2}\right) \\
\leqslant & 2\left\{\mathbf{P}\left(\sup _{t \leqslant T}\left|Z_{t}^{\varepsilon}\right|>\eta,\left\langle Z^{\varepsilon}\right\rangle_{T} \leqslant 2 l r^{2} \varepsilon^{2 \kappa}\right)\right. \\
& \left.\vee 2 \mathbf{P}\left(\sup _{0 \leqslant v \leqslant C}\left|\varepsilon^{1-\kappa} \int_{0}^{v / \varepsilon} \theta(s) d s\right|>r \varepsilon^{\kappa}\right)\right\} \\
\leqslant & 4 \exp \left(\frac{-\eta^{2}}{4 l r^{2} \varepsilon^{2 \kappa}}\right) \vee 4 \mathbf{P}\left(\sup _{0 \leqslant v \leqslant C}\left|\varepsilon^{1-2 \kappa} \int_{0}^{v / \varepsilon} \theta(s) d s\right|>r\right) .
\end{aligned}
$$

В силу произвольности $r$, участвующего в (5.8), получаем

$$
\begin{aligned}
& \varlimsup_{\varepsilon \rightarrow 0} \varepsilon^{2 \kappa} \ln \mathbf{P}\left(\sup _{t \leqslant T}\left|Z_{t}^{\varepsilon}\right|>\eta, \Omega \backslash \Upsilon_{C}^{\varepsilon}\right) \\
& \quad \leqslant \varepsilon^{2 \kappa} \ln 4-\frac{\eta^{2}}{4 l r^{2}} \vee \varlimsup_{\varepsilon \rightarrow 0} \varepsilon^{2 \kappa} \ln \mathbf{P}\left(\sup _{0<v \leqslant C}\left|\varepsilon^{1-2 \kappa} \int_{0}^{v / \varepsilon} \theta(s) d s\right|>r\right) \\
& \quad=-\frac{\eta^{2}}{4 l r^{2}} \underset{r \rightarrow 0}{\longrightarrow}-\infty
\end{aligned}
$$

где последнее равенство выполнено в силу (5.6).

Проверка второго условия в (1.4) производится аналогичным образом. Мы вводим стационарный процесс $\theta(t)=1-\mathbf{a} / \sigma^{2}(t)$, где константа $\mathbf{a}$ выбрана такой, чтобы $\mathbf{E} \theta(t)=0$, т.е. $\mathbf{a}=1 / \sum_{i=1}^{m}\left(\pi_{i} / a_{i}^{2}\right)$. Положим $H(x)=\int_{0}^{x} \int_{0}^{v} \theta(s) d s d v$. В соответствии с версией Крылова формулы Ито (см. [8]) получаем

$H\left(\frac{X_{t}^{\varepsilon}}{\varepsilon}\right)=H\left(\frac{x_{0}}{\varepsilon}\right)+\frac{1}{\varepsilon} \int_{0}^{t} \int_{0}^{X_{v}^{\varepsilon} / \varepsilon} \theta(s) d s d X_{v}^{\varepsilon}+\frac{1}{\varepsilon^{2(1-\kappa)}} \int_{0}^{t}\left[\sigma^{2}\left(\frac{X_{s}^{\varepsilon}}{\varepsilon}\right)-\mathbf{a}\right] d s$.

Отсюда

$$
\begin{aligned}
& \int_{0}^{t}\left[\sigma^{2}\left(\frac{X_{s}^{\varepsilon}}{\varepsilon}\right)-\mathbf{a}\right] d s=\varepsilon^{2(1-\kappa)} \int_{0}^{X_{t}^{\varepsilon} / \varepsilon} \int_{0}^{v} \theta(s) d s d v \\
& \quad-\varepsilon^{2(1-\kappa)} \int_{0}^{x_{0} / \varepsilon} \int_{0}^{v} \theta(s) d s d v-\varepsilon^{1-2 \kappa} \int_{0}^{t} \int_{0}^{X_{v}^{\varepsilon} / \varepsilon} \theta(s) d s b\left(\frac{X_{v}^{\varepsilon}}{\varepsilon}\right) d v
\end{aligned}
$$




$$
-\varepsilon^{1-\kappa} \int_{0}^{t} \int_{0}^{X_{v}^{\varepsilon} / \varepsilon} \theta(s) d s \sigma\left(\frac{X_{v}^{\varepsilon}}{\varepsilon}\right) d B_{v} .
$$

Остальная часть доказательства опускается, поскольку она полностью повторяет предшествующее доказательство.

6. Почему периодическая и случайная среды имеют разные режимы умеренных уклонений? Принцип умеренных уклонений в периодической и случайной средах неодинаков, в частности, для интервалов

$$
\underbrace{\kappa \in\left(0, \frac{1}{2}\right)}_{\text {период. ср. }} \text { и } \underbrace{\kappa \in\left(0, \frac{1}{6}\right)}_{\text {случ. ср. }} \text {. }
$$

Этот факт объясняется более быстрой гомогенизацией в периодической среде по сравнению со случайной средой. Поясним это наброском доказательства (1.4) в периодической среде.

Обозначим $\theta(s)$ любой из процессов $(b(s)-\mathbf{b}) / \sigma^{2}(s)$ или $1-\mathbf{a} / \sigma^{2}(s)$ и выберем $\mathbf{b}$ и а такими, чтобы

$$
\int_{0}^{1} \theta(s) d s=0
$$

T.e.

$$
\mathbf{b}=\int_{0}^{1} \frac{b(s) d s}{\sigma^{2}(s)} / \int_{0}^{1} \frac{d s}{\sigma^{2}(s)} \quad \text { и } \quad \mathbf{a}=1 / \int_{0}^{1} \frac{1}{\sigma^{2}(s)} d s .
$$

Положим $H(x)=\int_{0}^{x} \int_{0}^{v} \theta(s) d s d v$. По формуле Ито, примененной к $\varepsilon^{2(1-\kappa)} H\left(X_{t}^{\varepsilon} / \varepsilon\right)$, находим, что

$$
\begin{aligned}
\int_{0}^{t}\left[b\left(\frac{X_{s}^{\varepsilon}}{\varepsilon}\right)-\mathbf{b}\right] d s= & \int_{0}^{t}\left[\sigma^{2}\left(\frac{X_{s}^{\varepsilon}}{\varepsilon}\right)-\mathbf{a}\right] d s \\
= & \varepsilon^{2(1-\kappa)} \int_{0}^{X_{t}^{\varepsilon} / \varepsilon} \int_{0}^{v} \theta(s) d s d v \\
& -\varepsilon^{2(1-\kappa)} \int_{0}^{x_{0} / \varepsilon} \int_{0}^{v} \theta(s) d s d v \\
& -\varepsilon^{1-2 \kappa} \int_{0}^{t} \int_{0}^{X_{v}^{\varepsilon} / \varepsilon} \theta(s) d s b\left(\frac{X_{v}^{\varepsilon}}{\varepsilon}\right) d v \\
& -\varepsilon^{1-\kappa} \int_{0}^{t} \int_{0}^{X_{v}^{\varepsilon} / \varepsilon} \theta(s) d s \sigma\left(\frac{X_{v}^{\varepsilon}}{\varepsilon}\right) d B_{v} .
\end{aligned}
$$

Поскольку $\theta(s)$ - периодическая функция, (6.1) обеспечивает равномерную по $t \geqslant 0$ ограниченность величины $\left|\int_{0}^{t} \theta(s) d s\right|$. Это и есть основная причина быстрой гомогенизации. А именно, имеют место следующие оценки для величин, участвующих в (6.2), (6.3) и (6.4) (здесь $l$ - произвольная положительная константа):

$$
\varepsilon^{2(1-\kappa)}\left|\int_{0}^{X_{t}^{\varepsilon} / \varepsilon} \int_{0}^{v} \theta(s) d s d v\right| \leqslant l \varepsilon^{1-2 \kappa} \sup _{t \leqslant T}\left|X_{t}^{\varepsilon}\right|
$$




$$
\begin{aligned}
\varepsilon^{2(1-\kappa)}\left|\int_{0}^{x_{0} / \varepsilon} \int_{0}^{v} \theta(s) d s d v\right| & \leqslant l \varepsilon^{1-2 \kappa}\left|x_{0}\right|, \\
\varepsilon^{1-2 \kappa}\left|\int_{0}^{t} \int_{0}^{X_{v}^{\varepsilon} / \varepsilon} \theta(s) d s b\left(X_{v}^{\varepsilon} / \varepsilon\right) d v\right| & \leqslant l \varepsilon^{1-2 \kappa} .
\end{aligned}
$$

Вторая и третья оценки детерминированные. Поэтому две последние случайные величины являются экспоненциально плотными (с интенсивностью $\left.\varepsilon^{2 \kappa}\right)$ для любого $\kappa>0$. Поскольку $b$ и $\sigma$ ограничены, то, в силу неравенства (4.4),

$$
\lim _{C \rightarrow \infty} \varlimsup_{\varepsilon \rightarrow 0} \varepsilon^{2 \kappa} \ln \mathbf{P}\left(\sup _{t \leqslant T}\left|X_{t}^{\varepsilon}\right|>C\right)=-\infty
$$

и, значит, первая оценка также экспоненциально плотна (с интенсивностью $\left.\varepsilon^{2 \kappa}\right)$ для любого $\kappa>0$.

Ограничение на $\kappa$ вызвано требуемой экспоненциальной пренебрежимостью (с интенсивностью $\varepsilon^{2 \kappa}$ ) непрерывного мартингала

$$
Z_{t}^{\varepsilon}:=\varepsilon^{1-\kappa} \int_{0}^{t} \int_{0}^{X_{v}^{\varepsilon} / \varepsilon} \theta(s) d s \sigma\left(X_{v}^{\varepsilon} / \varepsilon\right) d B_{v} .
$$

Так как $\left\langle Z^{\varepsilon}\right\rangle_{t}=\varepsilon^{2(1-\kappa)} \int_{0}^{t}\left(\int_{0}^{X_{v}^{\varepsilon} / \varepsilon} \theta(s) d s\right)^{2} \sigma^{2}\left(X_{v}^{\varepsilon} / \varepsilon\right) d v$ и $\left|\int_{0}^{t} \theta(s) d s\right|$ равномерно ограничена по $t(!)$, то $\left\langle Z^{\varepsilon}\right\rangle_{T} \leqslant \varepsilon^{2(1-\kappa)} l$. Поэтому, в силу леммы 4.1 , $\mathbf{P}\left(\sup _{t \leqslant T}\left|Z_{t}^{\varepsilon}\right|>\eta\right)=\mathbf{P}\left(\sup _{t \leqslant T}\left|Z_{t}^{\varepsilon}\right|>\eta,\left\langle Z^{\varepsilon}\right\rangle_{T} \leqslant \varepsilon^{2(1-\kappa)} l\right) \leqslant 2 \exp \left(-\frac{\eta^{2}}{2 l \varepsilon^{2(1-\kappa)}}\right)$ и, следовательно, только при $\kappa<\frac{1}{2}$ имеет место требуемое соотношение:

$$
\varepsilon^{2 \kappa} \ln \mathbf{P}\left(\sup _{t \leqslant T}\left|Z_{t}^{\varepsilon}\right|>\eta\right) \leqslant \varepsilon^{2 \kappa} \ln 2-\frac{\eta^{2}}{2 l \varepsilon^{2(1-2 \kappa)}} \underset{\varepsilon \rightarrow 0}{\longrightarrow}-\infty .
$$

Приложение А. Принцип больших уклонений. Использование (1.4) существенно упрощает доказательство теоремы 3.1. Мы подчеркивали ранее, что импликация

$$
\text { (1.4) } \Rightarrow \text { утверждение теоремы } 3.1
$$

(см. [14, следствие 4.3.8], а также [13, следствие 6.7]) применима не только в нашем случае, но и для разнообразных классов семимартингалов.

Для удобства читателя мы показываем, как можно доказать (А.1) в нашем случае.

Пусть $X^{\varepsilon}=\left(X_{t}^{\varepsilon}\right)_{t \leqslant T}$ - непрерывный семимартингал, определенный на стохастическом базисе $\left(\Omega, \mathscr{F}, \mathbf{F}^{\varepsilon}=\left(\mathscr{F}_{t}^{\varepsilon}\right)_{t \leqslant T}, \mathbf{P}\right)$, удовлетворяющем стандартным условиям:

$$
X_{t}^{\varepsilon}=x_{0}+\int_{0}^{t} b_{s}^{\varepsilon} d s+\varepsilon^{\kappa} \int_{0}^{t} \alpha_{s}^{\varepsilon} d B_{s},
$$


где $B_{t}-$ броуновское движение, $b_{t}^{\varepsilon}$ и $\alpha_{t}^{\varepsilon}$ являются $\mathbf{F}^{\varepsilon}$-согласованными случайными процессами с $\int_{0}^{T}\left|b_{t}^{\varepsilon}\right| d t<\infty$ и $\int_{0}^{T}\left(\alpha_{s}^{\varepsilon}\right)^{2} d t<\infty$ п.н., $\varepsilon-$ малый параметр и $\kappa$ - положительное число.

Теорема А.1. Пусть $0<c_{1} \leqslant\left|\alpha_{s}^{\varepsilon}\right|^{2} \leqslant c_{2},\left|\beta_{s}^{\varepsilon}\right| \leqslant c_{3}$ и существуют константы $\mathbf{b} u \mathbf{a}>0$ такие, что для любого $\eta>0$

$$
\begin{aligned}
& \lim _{\varepsilon \rightarrow 0} \varepsilon^{2 \kappa} \ln \mathbf{P}\left(\sup _{t \leqslant T}\left|\int_{0}^{T}\left[b_{s}^{\varepsilon}-\mathbf{b}\right] d s\right|>\eta\right)=-\infty, \\
& \lim _{\varepsilon \rightarrow 0} \varepsilon^{2 \kappa} \ln \mathbf{P}\left(\sup _{t \leqslant T}\left|\int_{0}^{T}\left[\left(\alpha_{s}^{\varepsilon}\right)^{2}-\mathbf{a}\right] d s\right|>\eta\right)=-\infty .
\end{aligned}
$$

Тогда семейство $\left\{X^{\varepsilon}\right\}_{\varepsilon \rightarrow 0}$ допускает принцип больших уклонений в метрическом пространстве $\left(\mathbf{C}_{[0, T]}, \varrho\right)$ с параметром $\varepsilon^{2 \kappa}$ и функиионалом действия

$$
J(u)= \begin{cases}\frac{1}{2 \mathbf{a}} \int_{0}^{T}\left(\dot{u}_{t}-\mathbf{b}\right)^{2} d t, & u_{0}=x_{0}, d u_{t}=\dot{u}_{t} d t, \int_{0}^{T} \dot{u}_{t}^{2} d t<\infty \\ \infty & \text { в противном случае. }\end{cases}
$$

Доказательство этой теоремы использует стандартную импликацию (см., например, [10]):

$\left.\begin{array}{l}\text { Экспоненциальная плотность } \\ \text { Локальный принцип больших уклонений }\end{array}\right\} \Rightarrow$ Принцип больших уклонений.

Для проверки экспоненциальной плотности и локального принципа больших уклонений удобно использовать момент остановки

$$
\tau_{\varepsilon, \zeta}=\inf \left\{t \leqslant T:\left|\int_{0}^{t}\left[b_{s}^{\varepsilon}-\mathbf{b}\right] d s\right|+\left|\int_{0}^{t}\left[\left(\alpha_{s}^{\varepsilon}\right)^{2}-\mathbf{a}\right] d s\right|>\zeta\right\} .
$$

Заметим, что (А.2) и (А.3) влекут за собой $\lim _{\varepsilon \rightarrow 0} \varepsilon^{2 \kappa} \ln \mathbf{P}\left(\tau_{\varepsilon, \zeta}<\infty\right)=-\infty$ и, следовательно, для любого измеримого множества $\mathfrak{B}$

$$
\varlimsup_{\varepsilon \rightarrow 0} \varepsilon^{2 \kappa} \ln \mathbf{P}(\mathfrak{B}) \leqslant \varlimsup_{\zeta \rightarrow 0} \varlimsup_{\varepsilon \rightarrow 0} \varepsilon^{2 \kappa} \ln \mathbf{P}\left(\mathfrak{B} \cap\left\{\tau_{\varepsilon, \zeta}=\infty\right\}\right) .
$$

A.1. Экспоненциальная плотность. Следуя [10], мы будем доказывать, что

$\lim _{C \rightarrow \infty} \varlimsup_{\varepsilon \rightarrow 0} \varepsilon^{2 \kappa} \ln \mathbf{P}\left(\sup _{t \leqslant T}\left|X_{t}^{\varepsilon}\right|>C\right)=-\infty$

$\lim _{\delta \rightarrow 0} \varlimsup_{\varepsilon \rightarrow 0} \varepsilon^{2 \kappa} \ln \sup _{\theta<T} \mathbf{P}\left(\sup _{0<t \leqslant \delta}\left|X_{\theta+t}^{\varepsilon}-X_{\theta}^{\varepsilon}\right|>\eta\right)=-\infty \quad$ для всех $\eta>0$,

где $\theta$ является $\mathbf{F}^{\varepsilon}$-моментом остановки.

A.1.1. Доказательство (А.5). Пусть $\mathfrak{B}:=\left\{\sup _{t \leqslant T}\left|X_{t}^{\varepsilon}\right|>C\right\}$. В силу (А.4) достаточно доказать, что $\lim _{C \rightarrow \infty} \varlimsup_{\varepsilon \rightarrow 0} \varepsilon^{2 \kappa} \ln \mathbf{P}\left(\sup _{t \leqslant T}\left|X_{t}^{\varepsilon}\right|>\right.$ 
$\left.C, \tau_{\varepsilon, \zeta}=\infty\right)=-\infty$. С этой целью введем случайную величину $\left|x_{0}\right|+|\mathbf{b}| T+\zeta+\sup _{t \leqslant T}\left|\varepsilon^{\kappa} \int_{0}^{t \wedge \tau_{\varepsilon, \zeta}} \alpha_{s}^{\varepsilon} d B_{s}\right|$, являющуюся верхней оценкой $\sup _{t \leqslant T}\left|X_{t}^{\varepsilon}\right|$ на множестве $\left\{\tau_{\varepsilon, \zeta}=\infty\right\}$, и, тем самым, сведем доказательство к проверке соотношения

$$
\lim _{C \rightarrow \infty} \varlimsup_{\varepsilon \rightarrow 0} \varepsilon^{2 \kappa} \ln \mathbf{P}\left(\sup _{t \leqslant T}\left|\varepsilon^{\kappa} \int_{0}^{t \wedge \tau_{\varepsilon, \zeta}} \alpha_{s}^{\varepsilon} d B_{s}\right|>C, \tau_{\varepsilon, \zeta}=\infty\right)=-\infty .
$$

Применяя (4.4) к $M_{t}=\varepsilon^{\kappa} \int_{0}^{t \wedge \tau_{\varepsilon, \zeta}} \alpha_{s}^{\varepsilon} d B_{s}$ и принимая во внимание неравенство $\langle M\rangle_{T}=\varepsilon^{2 \kappa} \int_{0}^{T \wedge \tau_{\varepsilon, \zeta}}\left(\alpha_{s}^{\varepsilon}\right)^{2} d s \leqslant \varepsilon^{2 \kappa}[\mathbf{a} T+\zeta]$, получаем верхнюю границу $\mathbf{P}\left(\sup _{t \leqslant T}\left|M_{t}\right|>C\right) \leqslant 2 \exp \left(-C^{2} /\left(2 \varepsilon^{2 \kappa}(\mathbf{a} T+\zeta)\right)\right)$, которая дает (А.5).

A.1.2. Доказательство (А.6). Мы используем здесь тот же самый процесс $M_{t}$. Пусть $\mathbf{F}^{\varepsilon, \theta}=\left(\mathscr{F}_{\theta+t}^{\varepsilon}\right)_{t \geqslant 0}$. Обозначим $N_{t}^{\theta}:=M_{\theta+t}-M_{\theta}$. Случайный процесс $N_{t}^{\theta}$ является $\mathbf{F}^{\varepsilon, \theta}$-мартингалом с $\left\langle N^{\theta}\right\rangle_{t}=\varepsilon^{2 \kappa} \int_{\theta}^{\theta+t}\left(\alpha_{s}^{\varepsilon}\right)^{2} d s$. Введем $L_{t}^{\varepsilon}:=\int_{\theta}^{\theta+t} b_{s}^{\varepsilon} d s+N_{t}^{\theta}$ и заметим, что (А.6) эквивалентно условию

$$
\lim _{\zeta \rightarrow 0} \varlimsup_{\delta \rightarrow 0} \varlimsup_{\varepsilon \rightarrow 0} \varepsilon^{2 \kappa} \ln \sup _{\theta<T} \mathbf{P}\left(\sup _{0<t \leqslant \delta}\left|L_{t}^{\varepsilon}\right|>\eta\right)=-\infty .
$$

Тогда, в силу (А.4), достаточно доказать, что

$$
\lim _{\zeta \rightarrow 0} \varlimsup_{\delta \rightarrow 0} \varlimsup_{\varepsilon \rightarrow 0} \varepsilon^{2 \kappa} \ln \sup _{\theta<T} \mathbf{P}\left(\sup _{0<t \leqslant \delta}\left|L_{t}^{\varepsilon}\right|>\eta, \tau_{\varepsilon, \zeta}=\infty\right)=-\infty .
$$

Мы проверим это с помощью более сильного условия:

$$
\lim _{\zeta \rightarrow 0} \varlimsup_{\delta \rightarrow 0} \varlimsup_{\varepsilon \rightarrow 0} \varepsilon^{2 \kappa} \operatorname{lnsup}_{\theta<T} \mathbf{P}\left(\sup _{0<t \leqslant \delta \wedge \tau_{\varepsilon, \zeta}}\left|L_{t}^{\varepsilon}\right|>\eta\right)=-\infty .
$$

Поскольку $\sup _{0<t \leqslant \delta}\left|L_{t}^{\varepsilon}\right| \leqslant \delta|\mathbf{b}|+\zeta+\sup _{0<t \leqslant \delta}\left|N_{t \wedge \tau_{\varepsilon, \zeta}}^{\theta}\right|$, для фиксированного $\eta$ мы выбираем достаточно малые $\delta$ и $\zeta$ такие, что $\delta|\mathbf{b}|+\zeta<\eta$. При таком выборе вместо (А.7) достаточно доказать

$$
\lim _{\zeta \rightarrow 0} \varlimsup_{\delta \rightarrow 0} \varlimsup_{\varepsilon \rightarrow 0} \varepsilon^{2 \kappa} \ln _{\theta<T} \mathbf{P}\left(\sup _{0<t \leqslant \delta \wedge \tau_{\varepsilon, \zeta}}\left|N_{t}^{\theta}\right|>\eta-\delta|\mathbf{b}|-\zeta\right)=-\infty .
$$

Далее, поскольку $\left\langle N^{\theta}\right\rangle_{\delta \wedge \tau_{\varepsilon, \zeta}}=\varepsilon^{2 \kappa} \int_{\theta \wedge \tau_{\varepsilon, \zeta}+\delta \wedge \tau_{\varepsilon, \zeta}}\left(\alpha_{s}^{\varepsilon}\right)^{2} d s \leqslant \varepsilon^{2 \kappa}[\delta \mathbf{a}+\zeta]$, то, применяя (4.4) к $N_{t \wedge \tau_{\varepsilon, \zeta}}=\varepsilon^{\kappa} \int_{0}^{t \wedge \tau_{\varepsilon, \zeta}} \alpha_{s}^{\varepsilon} d B_{s}$, мы получаем следуюшую верхнюю оценку:

$$
\mathbf{P}\left(\sup _{t \leqslant \delta \wedge \tau_{\varepsilon, \zeta}}\left|N_{t}^{\theta}\right|>\eta-\delta|\mathbf{b}|-\zeta\right) \leqslant 2 \exp \left(-\frac{(\eta-\delta|\mathbf{b}|-\zeta)^{2}}{2 \varepsilon^{2 \kappa}(\delta \mathbf{a}+\zeta)}\right)
$$

которая гарантирует (А.6). 
A.2. Локальный принцип больших уклонений. Мы используем хорошо известный факт, что для экспоненциально плотного семейства $\left\{\left(X_{t}^{\varepsilon}\right)_{t \leqslant T}\right\}_{\varepsilon \rightarrow 0}$ условия

$$
\begin{aligned}
& \varlimsup_{\delta \rightarrow 0} \varlimsup_{\varepsilon \rightarrow 0} \varepsilon^{2 \kappa} \ln \mathbf{P}\left(\sup _{t \leqslant T}\left|X_{t}^{\varepsilon}-u_{t}\right| \leqslant \delta\right) \leqslant-J(u), \\
& \varliminf_{\delta \rightarrow 0} \varliminf_{\varepsilon \rightarrow 0} \varepsilon^{2 \kappa} \ln \mathbf{P}\left(\sup _{t \leqslant T}\left|X_{t}^{\varepsilon}-u_{t}\right| \leqslant \delta\right) \geqslant-J(u)
\end{aligned}
$$

определяют функционал действия $J(u), u \in \mathbf{C}_{[0, T]}$.

Поскольку $J(u) \in[0, \infty]$, мы вычисляем значения $J(u)$ для $u \in \mathfrak{U}_{1}$ и $\mathfrak{U}_{2}$, где

$$
\mathfrak{U}_{1}=\left\{u \in \mathbf{C}_{[0, T]}: J(u)<\infty\right\} \quad \text { и } \quad \mathfrak{U}_{2}=\left\{u \in \mathbf{C}_{[0, T]}: J(u)=\infty\right\} .
$$

A.2.1. Верхняя оченка для $u \in \mathfrak{U}_{1}$.

Лемма А.1. Неравенство (А.8) имеет место с $J(u)=(2 \mathbf{a})^{-1} \times$ $\int_{0}^{T}\left(\dot{u}_{t}-\mathbf{b}\right)^{2} d t$ для любого

$$
u \in\left\{u_{0}=x_{0}, d u_{t}=\dot{u}_{t} d t, \int_{0}^{T} \dot{u}_{t}^{2} d t<\infty\right\}=: \mathfrak{U}_{1} .
$$

Д ок аз а т е ль с т в о. Введем множество $\mathfrak{B}_{\delta}=\left\{\sup _{t \leqslant T} \mid X_{t}^{\varepsilon}-\right.$ $\left.u_{t} \mid \leqslant \delta\right\}$. Принимая во внимание (А.4), достаточно показать, что

$$
\varlimsup_{\zeta \rightarrow 0} \varlimsup_{\delta \rightarrow 0} \varlimsup_{\varepsilon \rightarrow 0} \varepsilon^{2 \kappa} \ln \mathbf{P}\left(\mathfrak{B}_{\delta} \cap\left\{\tau_{\varepsilon, \zeta}=\infty\right\}\right) \leqslant-\frac{1}{2 \mathbf{a}} \int_{0}^{T}\left(\dot{u}_{t}-\mathbf{b}\right)^{2} d t .
$$

Для этого используется экспоненциальный мартингал

$$
\mathfrak{z} t=\exp \left(\frac{1}{\varepsilon^{2 \kappa}}\left[\int_{0}^{T} \lambda(s)\left[d X_{s}^{\varepsilon}-b_{s}^{\varepsilon} d s\right]-\frac{1}{2} \int_{0}^{T} \lambda^{2}(s)\left(a_{s}^{\varepsilon}\right)^{2} d s\right]\right), \quad t \leqslant T,
$$

в котором $\lambda(s)$ - непрерывно дифференцируемая функция. Интегрируя по частям с помощью формулы Ито, получаем (здесь и далее $\dot{\lambda}(s)-$ производная $\lambda(s))$

$$
\int_{0}^{T} \lambda(s) d X_{s}^{\varepsilon}=\lambda(T) X_{T}^{\varepsilon}-\lambda(0) x_{0}-\int_{0}^{T} X_{s}^{\varepsilon} \dot{\lambda}(s) d s
$$

и записываем формулу для $\ln \mathfrak{z} t$ в следующем виде:

$\ln \mathfrak{z}_{T}=\frac{1}{\varepsilon^{2 \kappa}}\left[\lambda(T) X_{T}^{\varepsilon}-\lambda(0) x_{0}-\int_{0}^{T} X_{s}^{\varepsilon} \dot{\lambda}(s) d s-\int_{0}^{T}\left(\lambda(s) b_{s}^{\varepsilon}+\frac{\lambda^{2}(s)}{2}\left(a_{s}^{\varepsilon}\right)^{2}\right) d s\right]$.

Далее пишем

$$
\lambda(T) X_{T}^{\varepsilon}-\lambda(0) x_{0}-\int_{0}^{T} X_{s}^{\varepsilon} \dot{\lambda}(s) d s-\int_{0}^{T}\left(\lambda(s) b_{s}^{\varepsilon}+\frac{\lambda^{2}(s)}{2}\left(a_{s}^{\varepsilon}\right)^{2}\right) d s
$$




$$
\begin{aligned}
= & \lambda(T) u_{T}-\lambda(0) u_{0}-\int_{0}^{T} u_{s} \dot{\lambda}(s) d s-\int_{0}^{T}\left(\lambda(s) \mathbf{b}+\frac{\lambda^{2}(s)}{2} \mathbf{a}\right) d s \\
& +\lambda(T)\left[X_{t}^{\varepsilon}-u_{T}\right]-\int_{0}^{T}\left[X_{s}^{\varepsilon}-u_{s}\right] \dot{\lambda}(s) d s \\
& -\int_{0}^{T}\left(\lambda(s)\left[b_{s}^{\varepsilon}-\mathbf{b}\right]+\frac{\lambda^{2}(s)}{2}\left[\left(\alpha_{s}^{\varepsilon}\right)^{2}-\mathbf{a}\right]\right) d s .
\end{aligned}
$$

Интегрируя по частям, находим, что

$$
\begin{aligned}
\int_{0}^{T} \lambda(s)\left[b_{s}^{\varepsilon}-\mathbf{b}\right] d s= & \lambda(T) \int_{0}^{T}\left[b_{s}^{\varepsilon}-\mathbf{b}\right] d s-\int_{0}^{T} \dot{\lambda}(s) \int_{0}^{s}\left[b_{s^{\prime}}^{\varepsilon}-\mathbf{b}\right] d s^{\prime} d s \\
\int_{0}^{T} \frac{\lambda^{2}(s)}{2}\left[\left(a_{s}^{\varepsilon}\right)^{2}-\mathbf{a}\right] d s= & \frac{\lambda^{2}(T)}{2} \int_{0}^{T}\left[\left(a_{s}^{\varepsilon}\right)^{2}-\mathbf{a}\right] d s \\
& -\int_{0}^{T} \lambda(s) \dot{\lambda}(s) \int_{0}^{s}\left[\left(a_{s^{\prime}}^{\varepsilon}\right)^{2}-\mathbf{a}\right] d s^{\prime} d s
\end{aligned}
$$

и переписываем (А.10) следующим образом:

$$
\begin{aligned}
\lambda(T) & X_{T}^{\varepsilon}-\lambda(0) x_{0}-\int_{0}^{T} X_{s}^{\varepsilon} \dot{\lambda}(s) d s-\int_{0}^{T}\left(\lambda(s) b_{s}^{\varepsilon}+\frac{\lambda^{2}(s)}{2}\left(a_{s}^{\varepsilon}\right)^{2}\right) d s \\
= & \lambda(T) u_{T}-\lambda(0) u_{0}-\int_{0}^{T} u_{s} \dot{\lambda}(s) d s-\int_{0}^{T}\left(\lambda(s) \mathbf{b}+\frac{\lambda^{2}(s)}{2} \mathbf{a}\right) d s \\
& +\lambda(T)\left[X_{t}^{\varepsilon}-u_{T}\right]-\int_{0}^{T}\left[X_{s}^{\varepsilon}-u_{s}\right] \dot{\lambda}(s) d s \\
& -\lambda(T) \int_{0}^{T}\left[b_{s}^{\varepsilon}-\mathbf{b}\right] d s+\int_{0}^{T} \dot{\lambda}(s) \int_{0}^{s}\left[b_{s^{\prime}}^{\varepsilon}-\mathbf{b}\right] d s^{\prime} d s \\
& -\frac{\lambda^{2}(T)}{2} \int_{0}^{T}\left[\left(a_{s}^{\varepsilon}\right)^{2}-\mathbf{a}\right] d s+\int_{0}^{T} \lambda(s) \dot{\lambda}(s) \int_{0}^{s}\left[\left(a_{s^{\prime}}^{\varepsilon}\right)^{2}-\mathbf{a}\right] d s^{\prime} d s .
\end{aligned}
$$

Правая часть этого тождества оценивается снизу на множестве $\mathfrak{B}_{\delta} \cap$ $\left\{\tau_{\varepsilon, \zeta}=\infty\right\}$ величиной

$$
\begin{aligned}
\lambda(T) & u_{T}-\lambda(0) u_{0}-\int_{0}^{T} u_{s} \dot{\lambda}(s) d s-\int_{0}^{T}\left(\lambda(s) \mathbf{b}+\frac{\lambda^{2}(s)}{2} \mathbf{a}\right) d s \\
& -\delta\left(|\lambda(T)|+\int_{0}^{T}|\dot{\lambda}(s)| d s\right) \\
& -\zeta\left(|\lambda(T)|+\int_{0}^{T}|\dot{\lambda}(s)| d s+\frac{\lambda^{2}(T)}{2}+\int_{0}^{T}|\lambda(s) \dot{\lambda}(s)| d s\right) .
\end{aligned}
$$

Положим $l_{1}=|\lambda(T)|+\int_{0}^{T}|\dot{\lambda}(s)| d s+\lambda^{2}(T) / 2+\int_{0}^{T}|\lambda(s) \dot{\lambda}(s)| d s$. Тогда, учитывая тождество $\lambda(T) u_{T}-\lambda(0) u_{0}-\int_{0}^{T} u_{s} \dot{\lambda}(s) d s=\int_{0}^{T} \lambda(s) \dot{u}_{t} d t$, легко вывести следующую нижнюю оценку для $\mathfrak{z}_{T}$ :

$$
\mathfrak{z}_{*}=\exp \left(\frac{1}{\varepsilon^{2 \kappa}}\left[\int_{0}^{T}\left[\lambda(s)\left(\dot{u}_{s}-\mathbf{b}\right)-\frac{\lambda^{2}(s)}{2}\right] d s-l_{1}(\delta+\zeta)\right]\right) .
$$


Экспоненциальный мартингал $\mathfrak{z} t$, будучи положительным локальным мартингалом, является также супермартингалом. Поэтому $\mathbf{E}_{\mathfrak{z} T} \leqslant 1$. Эта оценка влечет за собой очевидную оценку: $\mathbf{E} I_{\left\{\mathfrak{B}_{\delta} \cap\left(\tau_{\varepsilon, \zeta}=\infty\right)\right\}} \mathfrak{z}_{*} \leqslant 1$. Вместе с (А.11) последняя оценка гарантирует

$$
\varlimsup_{\zeta \rightarrow 0} \varlimsup_{\delta \rightarrow 0} \varlimsup_{\varepsilon \rightarrow 0} \varepsilon^{2 \kappa} \ln \mathbf{P}\left(\mathfrak{B}_{\delta} \cap\left\{\tau_{\varepsilon, \zeta}=\infty\right\}\right) \leqslant-\int_{0}^{T}\left\{\lambda(s)\left[\dot{u}_{s}-\mathbf{b}\right]-\frac{\lambda^{2}(s)}{2} \mathbf{a}\right\} d s .
$$

Напомним, что соотношение (А.12) выведено при условии, что $\lambda(s)-$ непрерывно дифференцируемая функция. Предположим, что $\dot{u}_{s}-$ также непрерывно дифференцируемая функция. Тогда, выбирая $\lambda(s) \equiv$ $\left(\dot{u}_{s}-\mathbf{b}\right) / \mathbf{a}$, получаем требуемый результат. В общем случае $\dot{u}_{t}$, являясь лишь плотностью $u_{t}$ относительно $d t$, не обязана быть непрерывно дифференцируемой. В этом случае, поскольку $\int_{0}^{T}\left(\left(\dot{u}_{s}-\mathbf{b}\right) / \mathbf{a}\right)^{2} d s<\infty$, функцию $\lambda(s) \equiv\left(\dot{u}_{s}-\mathbf{b}\right) / \mathbf{a}$ можно аппроксимировать непрерывно дифференцируемыми функциями $\lambda_{m}(s), m \geqslant 1$ :

$$
\lim _{m \rightarrow \infty} \int_{0}^{T}\left(\lambda_{m}(s)-\frac{\dot{u}_{s}-\mathbf{b}}{\mathbf{a}}\right)^{2} d s=0 .
$$

Тогда

$$
\begin{aligned}
-\int_{0}^{T} & \left\{\lambda_{m}(s)\left[\dot{u}_{s}-\mathbf{b}\right]-\frac{\lambda_{m}^{2}(s)}{2} \mathbf{a}\right\} d s=-\int_{0}^{T} \frac{\left(\dot{u}_{s}-\mathbf{b}\right)^{2}}{2 \mathbf{a}} d s \\
+ & \frac{\mathbf{a}}{2} \int_{0}^{T}\left(\lambda_{m}(s)-\frac{\dot{u}_{s}-\mathbf{b}}{\mathbf{a}}\right)^{2} d s \underset{m \rightarrow \infty}{\longrightarrow}-\int_{0}^{T} \frac{\left(\dot{u}_{s}-\mathbf{b}\right)^{2}}{2 \mathbf{a}} d s .
\end{aligned}
$$

A.2.2. Верхняя оченка для $u \in \mathfrak{U}_{2}$. Принимая во внимание, что $\mathfrak{U}_{2}=$ $\mathbf{C}_{[0, T]} \backslash \mathfrak{U}_{1}$, достаточно установить верхние оценки в (А.8) при условиях:

(c.1) $u_{0}=x_{0}, d u_{t} \ll d t, \int_{0}^{T} \dot{u}^{2} d t=\infty$

(c.2) $u_{0}=x_{0}, d u_{t} \nless d t$,

(c.3) $u_{0} \neq x_{0}$.

Лемма А.2. Для любых и, удовлетворяющих условиям (c.1), (c.2) и (c.3), верхняя оченка в (А.8) имеет место с $J(u)=\infty$.

Д о к а з а т е ль с т в о. (c.1) В силу (А.12) и (А.4) для любой непрерывно дифференцируемой функции $\lambda(s)$ получаем

$$
\varlimsup_{\delta \rightarrow 0} \varlimsup_{\varepsilon \rightarrow 0} \varepsilon^{2 \kappa} \ln \mathbf{P}\left(\mathfrak{B}_{\delta}\right) \leqslant-\int_{0}^{T}\left\{\lambda(s)\left[\dot{u}_{s}-\mathbf{b}\right]-\frac{\lambda^{2}(s)}{2} \mathbf{a}\right\} d s .
$$

При каждом $n$ выбираем $\lambda_{n}(s)=\left(\left(\dot{u}_{s}-\mathbf{b}\right) / \mathbf{a}\right) I_{\left\{\left|\dot{u}_{s}\right| \leqslant n\right\}}$, а также последовательность непрерывно дифференцируемых функций, аппроксимирующих $\lambda_{n}(s)$ :

$$
\lim _{m \rightarrow \infty} \int_{0}^{T}\left[\lambda_{n}(s)-\lambda_{m, n}(s)\right]^{2} d s=0 .
$$


Для каждой функции $\lambda_{m, n}(s)$ неравенство (А.13) превращается в

$$
\varlimsup_{\delta \rightarrow 0} \varlimsup_{\varepsilon \rightarrow 0} \varepsilon^{2 \kappa} \ln \mathbf{P}\left(\mathfrak{B}_{\delta}\right) \leqslant-\int_{0}^{T}\left\{\lambda_{m, n}(s)\left[\dot{u}_{s}-\mathbf{b}\right]-\frac{\lambda_{m, n}^{2}(s)}{2} \mathbf{a}\right\} d s .
$$

Правая часть (А.14) стремится к $-\int_{0}^{T}\left\{\lambda_{n}(s)\left[\dot{u}_{s}-\mathbf{b}\right]-\lambda_{n}^{2}(s) \mathbf{a} / 2\right\} d s$ при $m \rightarrow \infty$, где $-\int_{0}^{T}\left\{\lambda_{n}(s)\left[\dot{u}_{s}-\mathbf{b}\right]-\lambda_{n}^{2}(s) \mathbf{a} / 2\right\} d s=-(2 \mathbf{a})^{-1} \int_{0}^{T}\left(\dot{u}_{s}-\right.$ b) $)^{2} I_{\left\{\left|\dot{u}_{s}\right| \leqslant n\right\}} d s$. Следовательно,

$$
\varlimsup_{\delta \rightarrow 0} \varlimsup_{\varepsilon \rightarrow 0} \varepsilon^{2 \kappa} \ln \mathbf{P}\left(\mathfrak{B}_{\delta}\right) \leqslant-\frac{1}{2 \mathbf{a}} \int_{0}^{T}\left(\dot{u}_{s}-\mathbf{b}\right)^{2} I_{\left\{\left|\dot{u}_{s}\right| \leqslant n\right\}} d s \underset{n \rightarrow \infty}{\longrightarrow}-\infty .
$$

(c.2) Принимая во внимание, что $d u_{t} \nless d t$, выберем последовательности $\left[s_{i}^{n}, t_{i}^{n}\right), i=1, \ldots n$, непересекающихся интервалов из отрезка $[0, T]$ такие, что

$$
\lim _{n \rightarrow \infty} \sum_{i=1}^{n} \frac{\left|u_{t_{i}^{n}}-u_{s_{i}^{n}}\right|^{2}}{t_{i}^{n}-s_{i}^{n}}=\infty
$$

Согласно неравенству Коши-Шварца-Буняковского, имеем

$$
\left(\sum_{i=1}^{n}\left|u_{t_{i}^{n}}-u_{s_{i}^{n}}\right|\right)^{2} \leqslant \sum_{i=1}^{n}\left[t_{i}^{n}-s_{i}^{n}\right] \cdot \sum_{i=1}^{n} \frac{\left|u_{t_{i}^{n}}-u_{s_{i}^{n}}\right|^{2}}{t_{i}^{n}-s_{i}^{n}} .
$$

Далее, для любой положительной малой величины $\gamma$ можно выбрать такие интервалы $\left[s_{i}, t_{i}\right), i=1, \ldots, n$, что $\sum_{i=1}^{n}\left[t_{i}^{n}-s_{i}^{n}\right] \leqslant \gamma$ для любого $n \geqslant n_{\gamma}$, где $n_{\gamma}$ - некоторое число, зависящее от $\gamma$, и в то же самое время $\sum_{i=1}^{n}\left|u_{t_{i}^{n}}-u_{s_{i}^{n}}\right| \geqslant D>0$. Теперь (А.16) влечет за собой неравенство $D^{2} / \gamma \leqslant \sum_{i=1}^{n}\left|u_{t_{i}^{n}}-u_{s_{i}^{n}}\right|^{2} /\left(t_{i}^{n}-s_{i}^{n}\right)$. Следовательно, (А.15) выполнено, поскольку $D$ остается положительным при $\gamma \rightarrow 0$.

Мы докажем теперь, что

$$
\begin{aligned}
\varlimsup_{\zeta \rightarrow 0} \varlimsup_{\delta \rightarrow 0} \varlimsup_{\varepsilon \rightarrow 0} \ln \mathbf{P}\left(\mathfrak{B}_{\delta} \cap\left\{\tau_{\varepsilon, \zeta}=\infty\right\}\right) \\
\leqslant-\frac{1}{2 \mathbf{a}}\left\{\mathbf{b}^{2} T-2 \mathbf{b}\left(u_{T}-u_{0}\right)+\sum_{i=1}^{n} \frac{\left[u_{t_{i}^{n}}-u_{s_{i}^{n}}\right]^{2}}{t_{i}^{n}-s_{i}^{n}}\right\},
\end{aligned}
$$

и применим (А.15).

Выбирая $\lambda(t)=\sum_{i} \lambda_{i} I_{\left\{s_{i}^{n} \leqslant t<t_{i}^{n}\right\}}$, введем экспоненциальный мартингал

$$
\mathfrak{z}_{t}=\exp \left(\frac{1}{\varepsilon^{2 \kappa}}\left[\int_{0}^{T} \lambda(s)\left[d X_{s}^{\varepsilon}-b_{s}^{\varepsilon} d s\right]-\frac{1}{2} \int_{0}^{T} \lambda^{2}(s)\left(a_{s}^{\varepsilon}\right)^{2} d s\right]\right)
$$

и оценим снизу значение $\mathfrak{z}_{T}$ на множестве $\mathfrak{B}_{\delta} \cap\left\{\tau_{\varepsilon, \zeta}=\infty\right\}$. Пишем

$$
\ln \mathfrak{z}_{T}=\frac{1}{\varepsilon^{2 \kappa}} \sum_{i=1}^{n}\left[\lambda_{i}\left[X_{t_{i}^{n}}^{\varepsilon}-X_{s_{i}^{n}}^{\varepsilon}\right]-\int_{s_{i}^{n}}^{t_{i}^{n}}\left\{\lambda_{i} b_{s}^{\varepsilon}+\frac{1}{2} \lambda_{i}^{2}\left(a_{s}^{\varepsilon}\right)^{2}\right\} d s\right]
$$




$$
\begin{aligned}
\geqslant & \frac{1}{\varepsilon^{2 \kappa}} \sum_{i=1}^{n}\left\{\lambda_{i}\left[u_{t_{i}^{n}}-u_{s_{i}^{n}}\right]-\left[\lambda_{i} \mathbf{b}+\frac{\lambda_{i}^{2}}{2} \mathbf{a}\right]\left(t_{i}^{n}-s_{i}^{n}\right)\right\} \\
& -\frac{1}{\varepsilon^{2 \kappa}} \sum_{i=1}^{n}\left(2\left|\lambda_{i}\right| \delta+\zeta\left[\left|\lambda_{i}\right|+\frac{\lambda_{i}^{2}}{2}\right]\right)\left(t_{i}^{n}-s_{i}^{n}\right):=\ln \mathfrak{z}_{*} .
\end{aligned}
$$

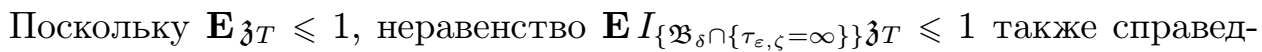
ливо и, значит, $\mathbf{E} I_{\left\{\mathfrak{B}_{\delta} \cap\left\{\tau_{\varepsilon, \zeta}=\infty\right\}\right\}} \mathfrak{z}_{*} \leqslant 1$. Этот результат влечет за собой

$$
\begin{aligned}
& \varlimsup_{\zeta \rightarrow 0} \varlimsup_{\delta \rightarrow 0} \varlimsup_{\varepsilon \rightarrow 0} \varepsilon^{2 \kappa} \ln \mathbf{P}\left(\mathfrak{B}_{\delta} \cap\left\{\tau_{\varepsilon, \zeta=\infty}\right\}\right) \\
& \quad \leqslant-\sum_{i=1}^{n}\left[\lambda_{i}\left(\left[u_{t_{i}^{n}}-u_{s_{i}^{n}}\right]-\mathbf{b}\left[t_{i}^{n}-s_{i}^{n}\right]\right)-\frac{\lambda_{i}^{2}}{2} \mathbf{a}\left[t_{i}^{n}-s_{i}^{n}\right]\right]
\end{aligned}
$$

и остается только выбрать $\lambda_{i}=\left(\left[u_{t_{i}^{n}}-u_{s_{i}^{n}}\right]-\mathbf{b}\left[t_{i}^{n}-s_{i}^{n}\right]\right) /\left(\mathbf{a}\left[t_{i}^{n}-s_{i}^{n}\right]\right)$.

(c.3) очевидно.

A.2.3. Нижняя оченка. Верхняя граница для локального принципа больших уклонений равна $-\infty$, если $u \in \mathfrak{U}_{2}$. Поэтому нижнюю границу надо проверять только для $u \in \mathfrak{U}_{1}$.

Лемма А.3. Пусть $u \in \mathfrak{U}_{1}$. Тогда (А.9) имеет место с $J(u)=$ $(2 \mathbf{a})^{-1} \int_{0}^{T}\left(\dot{u}_{t}-\mathbf{b}\right)^{2} d t$.

Д о к а з а т е ль с т в о. Достаточно установить, что

$$
\varliminf_{\delta \rightarrow 0} \varliminf_{\varepsilon \rightarrow 0} \varepsilon^{2 \kappa} \ln \mathbf{P}\left(\mathfrak{B}_{\delta}\right) \geqslant-\frac{1}{2 \mathbf{a}} \int_{0}^{T}\left[\dot{u}_{t}-\mathbf{b}\right]^{2} d t
$$

для любой функции $u$ с непрерывной второй производной $\ddot{u}_{t}$. Действительно, условие $\int_{0}^{T} \dot{u}_{t}^{2} d t<\infty$ позволяет выбрать последовательность функций $u_{m}(t), u_{m}(0) \equiv u_{0}$, с непрерывными вторыми производными $\left(\ddot{u}_{m}(t)\right)_{m \geqslant 1}$ таких, что

$$
\lim _{m \rightarrow \infty} \int_{0}^{T}\left(\dot{u}_{t}-\dot{u}_{m}(t)\right)^{2} d t=0 .
$$

Последнее свойство обеспечивает выполнение неравенства

$$
\sup _{t \leqslant T}\left|u_{t}-u_{m}(t)\right| \leqslant\left(T \int_{0}^{T}\left[\dot{u}_{s}-\dot{u}_{m}(s)\right]^{2} d s\right)^{1 / 2} \underset{m \rightarrow \infty}{\longrightarrow} 0
$$

Обозначим $\mathfrak{B}_{\delta, m}=\left\{\sup _{t \leqslant T}\left|X_{t}^{\varepsilon}-u_{m}(t)\right| \leqslant \delta\right\}$ и предположим, что нижняя оценка

$$
\varliminf_{\delta \rightarrow 0} \varliminf_{\varepsilon \rightarrow 0} \varepsilon^{2 \kappa} \ln \mathbf{P}\left(\mathfrak{B}_{\delta, m}\right) \geqslant-\frac{1}{2 \mathbf{a}} \int_{0}^{T}\left[\dot{u}_{m}(t)-\mathbf{b}\right]^{2} d t
$$

имеет место при каждом $m \geqslant 1$. 
Для $\gamma>\delta>0$ пишем

$$
\begin{aligned}
\mathfrak{B}_{\delta, m} & \subseteq\left(\mathfrak{B}_{\delta, m} \cap\left\{\sup _{t \leqslant T}\left|u_{t}-u_{m}(t)\right| \leqslant \gamma\right\}\right) \cup\left\{\sup _{t \leqslant T}\left|u_{t}-u_{m}(t)\right|>\gamma\right\} \\
& \subseteq\left\{\sup _{t \leqslant T}\left|X_{t}^{\varepsilon}-u_{t}\right| \leqslant 2 \gamma\right\} \cup\left\{\sup _{t \leqslant T}\left|u_{t}-u_{m}(t)\right|>\gamma\right\} .
\end{aligned}
$$

Поскольку при достаточно больших номерах $m$ множество $\left\{\sup _{t \leqslant T} \mid u_{t}-\right.$ $\left.u_{m}(t) \mid>\gamma\right\}$ оказывается пустым, имеет место оценка

$$
\mathbf{P}\left(\sup _{t \leqslant T}\left|X_{t}^{\varepsilon}-u_{t}\right| \leqslant 2 \gamma\right) \geqslant \mathbf{P}\left(\mathfrak{B}_{\delta, m}\right)
$$

и, значит,

$$
\begin{gathered}
\varliminf_{\varepsilon \rightarrow 0} \varepsilon^{2 \kappa} \ln \mathbf{P}\left(\sup _{t \leqslant T}\left|X_{t}^{\varepsilon}-u_{t}\right| \leqslant 2 \gamma\right) \geqslant \varliminf_{\delta \rightarrow 0} \frac{\lim _{\varepsilon \rightarrow 0}}{\varepsilon^{2 \kappa}} \ln \mathbf{P}\left(\mathfrak{B}_{\delta, m}\right) \\
\geqslant-\frac{1}{2 \mathbf{a}} \int_{0}^{T}\left[\dot{u}_{m}(t)-\mathbf{b}\right]^{2} d t \underset{m \rightarrow \infty}{\longrightarrow}-\frac{1}{2 \mathbf{a}} \int_{0}^{T}\left[\dot{u}_{t}-\mathbf{b}\right]^{2} d t,
\end{gathered}
$$

т.е. остается только перейти к пределу $\underline{\lim }_{\gamma \rightarrow 0}$ в левой части полученного неравенства.

Мы воспользуемся также тем фактом, что (А.17) с функцией $u$, обладающей непрерывной $\ddot{u}$, выполняется при условии, что

$$
\varliminf_{\zeta \rightarrow 0} \varliminf_{\delta \rightarrow \infty} \varliminf_{\varepsilon \rightarrow 0} \varepsilon^{2 \kappa} \ln \mathbf{P}\left(\sup _{t \leqslant T \wedge \tau_{\varepsilon, \zeta}}\left|X_{t}^{\varepsilon}-u_{t}\right| \leqslant \delta\right) \geqslant-\frac{1}{2 \mathbf{a}} \int_{0}^{T}\left[\dot{u}_{t}-\mathbf{b}\right]^{2} d t .
$$

Теперь с помощью очевидного включения

$$
\begin{aligned}
& \left\{\sup _{t \leqslant T \wedge \tau_{\varepsilon, \zeta}}\left|X_{t}^{\varepsilon}-u_{t}\right| \leqslant \delta\right\}=\left\{\sup _{t \leqslant T}\left|X_{t}^{\varepsilon}-u_{t}\right| \leqslant \delta, \tau_{\varepsilon, \zeta}=\infty\right\} \\
& \cup\left\{\sup _{t \leqslant T \wedge \tau_{\varepsilon, \zeta}}\left|X_{t}^{\varepsilon}-u_{t}\right| \leqslant \delta, \tau_{\varepsilon, \zeta}<\infty\right\} \subseteq\left\{\sup _{t \leqslant T}\left|X_{t}^{\varepsilon}-u_{t}\right| \leqslant \delta\right\} \cup\left\{\tau_{\varepsilon, \zeta}<\infty\right\}
\end{aligned}
$$

получаем неравенство

$$
\mathbf{P}\left(\sup _{t \leqslant T \wedge \tau_{\varepsilon, \zeta}}\left|X_{t}^{\varepsilon}-u_{t}\right| \leqslant \delta\right) \leqslant 2\left[\mathbf{P}\left(\sup _{t \leqslant T}\left|X_{t}^{\varepsilon}-u_{t}\right| \leqslant \delta\right) \vee \mathbf{P}\left(\tau_{\varepsilon, \zeta}<\infty\right)\right],
$$

из которого (А.17) следует в силу (А.18) и $\overline{\lim }_{\zeta \rightarrow \infty} \varlimsup_{\varepsilon \rightarrow 0} \varepsilon^{2 \kappa} \ln \mathbf{P}\left(\tau_{\varepsilon, \zeta}<\right.$ $\infty)=-\infty$.

Начиная с этого момента мы остановимся на доказательстве (А.18) для $u$ с непрерывной второй производной. Введем экспоненциальный мартингал $\mathfrak{z}_{t}=\exp \left\{M_{t}-\frac{1}{2}\langle M\rangle_{t}\right\}$, где

$$
M_{t}=\frac{1}{\varepsilon^{\kappa}} \int_{0}^{t \wedge \tau_{\varepsilon, \zeta}} \frac{\dot{u}_{t}-\beta_{s}^{\varepsilon}}{\alpha_{s}^{\varepsilon}} d B_{s} \quad \text { и } \quad\langle M\rangle_{t}=\frac{1}{\varepsilon^{2 \kappa}} \int_{0}^{t \wedge \tau_{\varepsilon, \zeta}} \frac{\left(\dot{u}_{t}-\beta_{s}^{\varepsilon}\right)^{2}}{\left(\alpha_{t}^{\varepsilon}\right)^{2}} d t .
$$

Напомним, что $\beta_{s}^{\varepsilon}$ ограничена сверху, а $\left(\alpha_{s}^{\varepsilon}\right)^{2}$ строго положительна. Поэтому ограниченность $\dot{u}_{t}$ на $[0, T]$ обеспечивает ограниченность $\langle M\rangle_{T}$. 
Отсюда $\mathbf{E}_{\mathfrak{z}_{T}}=1$. Положим $d \overline{\mathbf{P}}=\mathfrak{z}_{T} d \mathbf{P}$. Благодаря тому, что $\mathfrak{z}_{T}>0$ $\mathbf{P}$-п.н., меры $\overline{\mathbf{P}}$ и $\mathbf{P}$ эквивалентны с $d \mathbf{P}=\mathfrak{z}_{T}^{-1} d \overline{\mathbf{P}}$. Поэтому

$$
\mathbf{P}\left(\sup _{t \leqslant T \wedge \tau_{\varepsilon, \zeta}}\left|X_{t}^{\varepsilon}-u_{t}\right| \leqslant \delta\right)=\int_{\left\{\sup _{t \leqslant T \wedge \tau_{\varepsilon, \zeta}}\left|X_{t}^{\varepsilon}-u_{t}\right| \leqslant \delta\right\}} \mathfrak{z}_{T}^{-1} d \overline{\mathbf{P}} .
$$

Покажем сначала, что

$$
\varliminf_{\zeta \rightarrow 0} \varliminf_{\varepsilon \rightarrow 0} \overline{\mathbf{P}}\left(\sup _{t \leqslant T \wedge \tau_{\varepsilon, \zeta}}\left|X_{t}^{\varepsilon}-u_{t}\right| \leqslant \delta\right)=1 .
$$

По теореме Гирсанова (см., например, $[12$, теорема $2, \S 5$, гл. 4]) случайный процесс

$$
\bar{B}_{t}=B_{t}-\frac{1}{\varepsilon^{\kappa}} \int_{0}^{t \wedge \tau_{\varepsilon, \zeta}} \frac{\dot{u}_{s}-\beta_{s}^{\varepsilon}}{\alpha_{s}^{\varepsilon}} d s
$$

является броуновским движением относительно меры $\overline{\mathbf{P}}$. Оба процесса $X_{t}^{\varepsilon}$ и $\mathfrak{z} t$ являются семимартингалами относительно $\overline{\mathbf{P}}$. В частности,

$$
X_{t \wedge \tau_{\varepsilon, \zeta}}^{\varepsilon}=x_{0}+\int_{0}^{t \wedge \tau_{\varepsilon, \zeta}} \dot{u}_{s} d s+\varepsilon^{\kappa} \int_{0}^{t \wedge \tau_{\varepsilon, \zeta}} \alpha_{s}^{\varepsilon} d \bar{B}_{s}=u_{t \wedge \tau_{\varepsilon, \zeta}}+\varepsilon^{\kappa} \int_{0}^{t \wedge \tau_{\varepsilon, \zeta}} \alpha_{s}^{\varepsilon} d \bar{B}_{s} .
$$

Следовательно,

$$
\sup _{t \leqslant T \wedge \tau_{\varepsilon, \zeta}}\left|X_{t}^{\varepsilon}-u_{t}\right|=\sup _{t \leqslant T}\left|\varepsilon^{\kappa} \int_{0}^{t \wedge \tau_{\varepsilon, \zeta}} \alpha_{s}^{\varepsilon} d \bar{B}_{s}\right| .
$$

Теперь с помощью неравенства Дуба получаем

$$
\begin{gathered}
\overline{\mathbf{P}}\left(\sup _{t \leqslant T}\left|\varepsilon^{\kappa} \int_{0}^{t \wedge \tau_{\varepsilon, \zeta}} \alpha_{s}^{\varepsilon} d \bar{B}_{s}\right| \leqslant \delta\right)=1-\overline{\mathbf{P}}\left(\sup _{t \leqslant T}\left|\varepsilon^{\kappa} \int_{0}^{t \wedge \tau_{\varepsilon, \zeta}} \alpha_{s}^{\varepsilon} d \bar{B}_{s}\right|>\delta\right) \\
\geqslant 1-\frac{4 \varepsilon^{2 \kappa}}{\delta^{2}} \overline{\mathbf{E}} \int_{0}^{T \wedge \tau_{\varepsilon, \zeta}}\left(\alpha_{s}^{\varepsilon}\right)^{2} d s \geqslant 1-\frac{4 \varepsilon^{2 \kappa}}{\delta^{2}}[\mathbf{a} T+\zeta] \underset{\varepsilon \rightarrow 0}{\longrightarrow} 1 .
\end{gathered}
$$

Возвратимся теперь к анализу (А.19). В соответствии с (А.20)

$$
\mathfrak{z}_{T}=\exp \left(\frac{1}{\varepsilon^{\kappa}} \int_{0}^{T \wedge \tau_{\varepsilon, \zeta}} \frac{\dot{u}_{t}-\beta_{s}^{\varepsilon}}{\alpha_{s}^{\varepsilon}} d \bar{B}_{s}+\frac{1}{2 \varepsilon^{2 \kappa}} \int_{0}^{T \wedge \tau_{\varepsilon, \zeta}} \frac{\left[\dot{u}_{t}-\beta_{s}^{\varepsilon}\right]^{2}}{\left(\alpha_{s}^{\varepsilon}\right)^{2}} d s\right) .
$$

Обозначим $\mathfrak{B}_{\delta, \varepsilon}:=\left\{\sup _{t \leqslant T \wedge \tau_{\varepsilon, \zeta}}\left|X_{t}^{\varepsilon}-u_{t}\right| \leqslant \delta\right\}$. Тогда (А.19) можно представить в следующем виде:

$$
\begin{aligned}
\mathbf{P}\left(\mathfrak{B}_{\delta, \varepsilon}\right)=\int_{\mathfrak{B}_{\delta, \varepsilon}} \exp ( & -\frac{1}{\varepsilon^{2 \kappa}} \int_{0}^{T \wedge \tau_{\varepsilon, \zeta}} \frac{\dot{u}_{t}-\beta_{s}^{\varepsilon}}{\alpha_{s}^{\varepsilon}} d \bar{B}_{s} \\
& \left.-\frac{1}{2 \varepsilon^{2 \kappa}} \int_{0}^{T \wedge \tau_{\varepsilon, \zeta}} \frac{\left[\dot{u}_{s}-\beta_{s}^{\varepsilon}\right]^{2}}{\left(\alpha_{s}^{\varepsilon}\right)^{2}} d s\right) d \overline{\mathbf{P}} .
\end{aligned}
$$

Далее мы используем несколько очевидных соотношений. Выберем $l$ в качестве верхней границы для

$$
\frac{\left(\dot{u}_{s}-\mathbf{b}\right)^{2}}{\mathbf{a}\left(\alpha_{s}^{\varepsilon}\right)^{2}}+\frac{2\left|\dot{u}_{s}-\mathbf{b}\right|+\left|\mathbf{b}-\beta_{s}^{\varepsilon}\right|}{\mathbf{a}}\left[1+\frac{\left|\mathbf{a}-\left(\alpha_{s}^{\varepsilon}\right)^{2}\right|}{\left(\alpha_{s}^{\varepsilon}\right)^{2}}\right] .
$$


Пишем

$$
\begin{aligned}
\int_{0}^{T \wedge \tau_{\varepsilon, \zeta}} & \frac{\left[\dot{u}_{s}-\beta_{s}^{\varepsilon}\right]^{2}}{\left(\alpha_{s}^{\varepsilon}\right)^{2}} d s=\int_{0}^{T \wedge \tau_{\varepsilon, \zeta}} \frac{\left[\left(\dot{u}_{s}-\mathbf{b}\right)+\left(\mathbf{b}-\beta_{s}^{\varepsilon}\right)\right]^{2}}{\mathbf{a}}\left[1+\frac{\mathbf{a}-\left(\alpha_{s}^{\varepsilon}\right)^{2}}{\left(\alpha_{s}^{\varepsilon}\right)^{2}}\right] d s \\
\leqslant & \int_{0}^{T \wedge \tau_{\varepsilon, \zeta}} \frac{\left(\dot{u}_{s}-\mathbf{b}\right)^{\mathbf{2}}}{\mathbf{a}} d s+\int_{0}^{T \wedge \tau_{\varepsilon, \zeta}} \frac{\left(\dot{u}_{s}-\mathbf{b}\right)^{2}}{\mathbf{a}} \frac{\left|\mathbf{a}-\left(\alpha_{s}^{\varepsilon}\right)^{2}\right|}{\left(\alpha_{s}^{\varepsilon}\right)^{2}} d s \\
& +\int_{0}^{T \wedge \tau_{\varepsilon, \zeta}} \frac{2\left|\dot{u}_{s}-\mathbf{b}\right|\left|\mathbf{b}-\beta_{s}^{\varepsilon}\right|+\left(\mathbf{b}-\beta_{s}^{\varepsilon}\right)^{2}}{\mathbf{a}}\left[1+\frac{\left|\mathbf{a}-\left(\alpha_{s}^{\varepsilon}\right)^{2}\right|}{\left(\alpha_{s}^{\varepsilon}\right)^{2}}\right] d s \\
\leqslant & \int_{0}^{T \wedge \tau_{\varepsilon, \zeta}} \frac{\left(\dot{u}_{s}-\mathbf{b}\right)^{\mathbf{2}}}{\mathbf{a}} d s+l \int_{0}^{T \wedge \tau_{\varepsilon, \zeta}}\left[\left|\mathbf{a}-\left(\alpha_{s}^{\varepsilon}\right)^{2}\right|+\left|\mathbf{b}-\beta_{s}^{\varepsilon}\right|\right] d s \\
\leqslant & \int_{0}^{T \wedge \tau_{\varepsilon, \zeta}} \frac{\left(\dot{u}_{s}-\mathbf{b}\right)^{\mathbf{2}}}{\mathbf{a}} d s+\zeta l .
\end{aligned}
$$

Полученная оценка влечет за собой

$$
\begin{aligned}
\int_{\mathfrak{B}_{\delta, \varepsilon}} & \exp \left(-\frac{1}{\varepsilon^{\kappa}} \int_{0}^{T \wedge \tau_{\varepsilon, \zeta}} \frac{\dot{u}_{s}-\beta_{s}^{\varepsilon}}{\alpha_{s}^{\varepsilon}} d \bar{B}_{s}-\frac{1}{2 \varepsilon^{2 \kappa}} \int_{0}^{T \wedge \tau_{\varepsilon, \zeta}} \frac{\left[\dot{u}_{s}-\beta_{s}^{\varepsilon}\right]^{2}}{\left(\alpha_{s}^{\varepsilon}\right)^{2}} d s\right) d \overline{\mathbf{P}} \\
\geqslant & \exp \left(-\frac{1}{2 \varepsilon^{2 \kappa}} \int_{0}^{T} \frac{\left(\dot{u}_{s}-\mathbf{b}\right)^{2}}{\mathbf{a}} d s-\frac{\zeta l}{2 \varepsilon^{2 \kappa}}\right) \\
& \times \int_{\mathfrak{B}_{\delta, \zeta}} 1 \wedge \exp \left(-\frac{1}{\varepsilon^{\kappa}} \int_{0}^{T \wedge \tau_{\varepsilon, \zeta}} \frac{\dot{u}_{t}-\beta_{s}^{\varepsilon}}{\left(\alpha_{s}^{\varepsilon}\right)^{2}} d \bar{B}\right) d \overline{\mathbf{P}}
\end{aligned}
$$

$(\ll 1 \wedge \exp (\ldots) \gg$ вводится только для того, чтобы иметь ограниченную экспоненту в последнем интеграле).

Итак, мы получаем следующую нижнюю границу:

$$
\begin{aligned}
\mathbf{P}^{\varepsilon^{2 \kappa}}\left(\mathfrak{B}_{\delta, \varepsilon}\right) \geqslant & \exp \left(-\frac{1}{2 \mathbf{a}} \int_{0}^{T}\left(\dot{u}_{s}-\mathbf{b}\right)^{2} d t-\frac{\zeta l}{2}\right) \\
& \times\left(\int_{\mathfrak{B}_{\delta, \varepsilon}} 1 \wedge \exp \left(-\frac{1}{\varepsilon^{\kappa}} \int_{0}^{T \wedge \tau_{\varepsilon, \zeta}} \frac{\dot{u}_{s}-\beta_{s}^{\varepsilon}}{\left(\alpha_{s}^{\varepsilon}\right)^{2}} d \bar{B}\right) d \overline{\mathbf{P}}\right)^{\varepsilon^{2 \kappa}},
\end{aligned}
$$

и по неравенству Гёльдера (здесь $\varepsilon<1$ )

$$
\begin{aligned}
& \int_{\mathfrak{B}_{\delta, \varepsilon}} 1 \\
& \leqslant\left(\exp \left(-\varepsilon^{\kappa} \int_{0}^{T \wedge \tau_{\varepsilon, \zeta}} \frac{\dot{u}_{s}-\beta_{s}^{\varepsilon}}{\left(\alpha_{s}^{\varepsilon}\right)^{2}} d \bar{B}_{s}\right) d \overline{\mathbf{P}}\right. \\
& \leqslant\left(\int_{\mathfrak{B}_{\delta, \varepsilon}} 1 \wedge \exp \left(-\frac{1}{\varepsilon^{\kappa}} \int_{0}^{T \wedge \tau_{\varepsilon, \zeta}} \frac{\dot{u}_{s}-\beta_{s}^{\varepsilon}}{\left(\alpha_{s}^{\varepsilon}\right)^{2}} d \bar{B}_{s}\right) d \overline{\mathbf{P}}\right)^{\varepsilon^{2 \kappa}} .
\end{aligned}
$$

Таким образом,

$$
\begin{aligned}
\varliminf_{\varepsilon \rightarrow 0} & \varepsilon^{2 \kappa} \ln \mathbf{P}\left(\sup _{t \leqslant T \wedge \tau_{\varepsilon, \zeta}}\left|X_{t}^{\varepsilon}-u_{t}\right| \leqslant \delta\right) \geqslant-\frac{1}{2 \mathbf{a}} \int_{0}^{T}\left(\dot{u}_{s}-\mathbf{b}\right)^{2} d s \\
& -\frac{\zeta l}{2}+\varliminf_{\varepsilon \rightarrow 0} \varepsilon^{2 \kappa} \ln \int_{\mathfrak{B}_{\delta, \varepsilon}} 1 \wedge \exp \left(-\varepsilon^{\kappa} \int_{0}^{T \wedge \tau_{\varepsilon, \zeta}} \frac{\dot{u}_{s}-\beta_{s}^{\varepsilon}}{\left(\alpha_{s}^{\varepsilon}\right)^{2}} d \bar{B}_{s}\right) d \overline{\mathbf{P}} \\
\geqslant & -\frac{1}{2 \mathbf{a}} \int_{0}^{T}\left(\dot{u}_{s}-\mathbf{b}\right)^{2} d s-\frac{\zeta l}{2}+\ln \frac{\lim _{\varepsilon \rightarrow 0}}{\int_{\mathfrak{B}_{\delta, \varepsilon}} \psi^{\varepsilon} d \overline{\mathbf{P}},}
\end{aligned}
$$


где $\psi^{\varepsilon}:=1 \wedge \exp \left(-\varepsilon^{\kappa} \int_{0}^{T \wedge \tau_{\varepsilon, \zeta}}\left(\left(\dot{u}_{s}-\beta_{s}^{\varepsilon}\right) /\left(\alpha_{s}^{\varepsilon}\right)^{2}\right) d \bar{B}_{s}\right)$. В силу (А.21), $\lim _{\varepsilon \rightarrow 0} \overline{\mathbf{P}}\left(\mathfrak{B}_{\delta, \varepsilon}\right)=1$ и, кроме того, $\lim _{\varepsilon \rightarrow 0} \int_{\Omega} \psi^{\varepsilon} d \overline{\mathbf{P}}=1$. Отсюда, благодаря $\psi^{\varepsilon} \leqslant 1$, получаем

$$
\int_{\mathfrak{B}_{\delta, \varepsilon}} \psi^{\varepsilon} d \overline{\mathbf{P}}=\int_{\Omega} \psi^{\varepsilon} d \overline{\mathbf{P}}-\int_{\Omega \backslash \mathfrak{B}_{\delta, \varepsilon}} \psi^{\varepsilon} d \overline{\mathbf{P}} \geqslant \int_{\Omega} \psi^{\varepsilon} d \overline{\mathbf{P}}-\int_{\Omega \backslash \mathfrak{B}_{\delta, \varepsilon}} d \overline{\mathbf{P}} \underset{\varepsilon \rightarrow 0}{\longrightarrow} 1,
$$

т.е. $\ln \underline{\lim }_{\varepsilon \rightarrow 0} \int_{\mathfrak{B}_{\delta, \varepsilon}} \psi^{\varepsilon} d \overline{\mathbf{P}} \geqslant 0$.

В силу произвольности $\zeta$, (А.17) выполнено.

\section{СПИСОК ЛИТЕРАТУРЫ}

1. Dembo A., Zeitouni O. Large Deviations Techniques and Applications. New York: Springer-Verlag, 1998, $396 \mathrm{p}$.

2. Brox T. A one-dimensional diffusion process in a Wiener medium. - Ann. Probab., 1986, v. 14, № 4, p. 1206-1218.

3. Dupuis P., Ellis R.S. A Weak Convergence Approach to the Theory of Large Deviations. New York: Wiley, 1997, 479 p.

4. Freidlin M.I., Sowers R.B. A comparison of homogenization and large deviations, with applications to wavefront propagation. - Stochastic Process. Appl., 1999, v. 82, № 1 , p. $23-52$.

5. Вентиель А.Д., Фрейдлин М. И. Флуктуация в динамических системах под действием малых случайных возмущений. М.: Наука, 1979, 424 с.

6. Гертнер Ю., Фрейдлин М. И. О распространении концентрационных волн в периодических и случайных средах. - Докл. АН СССР, 1979, т. 249, № 3, с. 521-525.

7. Жакод Ж., Ширяев А. Н. Предельные теоремы для случайных процессов, т. 1, 2. М.: Наука, 1994, 542 с., 366 с.

8. Крылюв Н. В. Управляемые процессы диффузионного типа. М.: Наука, 1977, $399 \mathrm{c}$.

9. Liptser R.S., Spokoiny V. Moderate deviations type evaluation for integral functionals of diffusion processes. - Electron. J. Probab., 1999, v. 4, № 17, p. 1-25.

10. Liptser R.S., Pukhalskii A. A. Limit theorems on large deviations for semimartingales. - Stochastics Stochastics Rep., 1992, v. 38, № 4, p. 201-249.

11. Liptser R. S., Shiryaev A. N. Statistics of Random Processes. I. General theory. Berlin: Springer-Verlag, 2001, 427 p.

12. Липчер Р. Ш., Ширяев А. Н. Теория мартингалов. М.: Наука, 1986, 512 с.

13. Puhalskii A. A. Large deviations of semimartingales via convergence of the predictable characteristics. - Stochastics Stochastics Rep., 1994, v. 49, № 1-2, p. 27-85.

14. Puhalskii A.A. Large Deviations and Idempotent Pobability. Boca Raton: Chapman \& Hall/CRC, 2001, 500 p.

15. Schumacher S. Diffusions with random coefficients. - Particle Systems, Random Media and Large Deviations (Brunswick, Maine, 1984). Providence: Amer. Math. Soc., 1985, p. 351-356. (Contemp. Math., v. 41.)

Поступила в редакцию

17.III. 2007

Исправленный вариант

12.X.2008 